\title{
A new monolithic Newton-multigrid-based FEM solution scheme for large strain dynamic poroelasticity problems
}

\author{
A. Obaid ${ }^{1, *}$, St. Turek ${ }^{1}$, Y. Heider ${ }^{2}$ and B. Markert ${ }^{2}$ \\ ${ }^{1}$ Institute of Applied Mathematics (LS3), TU Dortmund university, Vogelpothsweg 87, 44227 Dortmund, Germany \\ ${ }^{2}$ Institute of General Mechanics (IAM), RWTH Aachen University, 52062 Aachen, Germany
}

\begin{abstract}
SUMMARY
This paper presents a new efficient monolithic finite element solution scheme to treat the set of PDEs governing a two-dimensional, biphasic, saturated TPM model with intrinsically coupled and incompressible solid and fluid constituents for infinitesimal and large elastic deformation. Our approach, which inherits some of its techniques from CFD, is characterized by the following aspects: (1) It only performs operator evaluation with no additional Gateaux derivatives. In particular, the computations of the time-consuming material tangent matrix are not involved here; (2) It solves the non-linear dynamic problem with no restriction on the strength of coupling; (3) It is more efficient than the linear uv $p$ solver discussed in previous works; (4) It requires weaker derivatives and hence lower order FE can be tested; (5) The boundary conditions are reduced, solution independent and more convenient to apply than in the old uv $p$ formulation. For the purpose of validation and comparison, prototypical simulations including analytical solutions are carried out and at the end, an adaptive time stepping (ATS) procedure is introduced to handle the rapid change in the numbers of non-linear iterations that may occur.
\end{abstract}

KEY WORDS: Theory of Porous Media (TPM); Updated Lagrangian (UL); Adaptive Time Stepping (ATS); Finite Element Method (FEM); Computational Fluid Dynamics (CFD); Picard Iteration Method; Hyperelasticity;

\section{INTRODUCTION}

The theoretical and numerical prediction of fluid-saturated porous solid behavior subjected to dynamic or quasi-static loadings is a challenging and important problem in different fields of engineering, such as in Geo-mechanics and Biomechanics, see, e. g., Ref. [1, 2, 3, 4]. The behavior of such materials is mainly governed by the mutual interaction between the solid skeleton and the pore fluid, where this interaction can be classified as a strong coupling especially in dynamic problems, see Ref. [5, 6].

The material description of the fluid-saturated heterogeneous porous solids is introduced within a thermodynamically consistent continuum mechanics framework using the Theory of Porous Media (TPM). In the framework of the TPM, a macroscopic description of general immiscible multi-phasic aggregates is introduced, where the individual constituents (here: solid and fluid) are considered to be in a state of ideal disarrangement over a homogenized representative volume element (RVE) in the sense of superimposed and interacting continua, cf. Ref. [7, 8] for detailed discussions. With regard to saturated porous media dynamics, the theoretical and numerical procedures have

* Correspondence to: Abdulrahman Obaid, Institute of Applied Mathematics (LS3), TU Dortmund university, Vogelpothsweg 87, 44227 Dortmund, Germany. Email: aobaid@math.tu-dortmund.de, Tel.: +492317553075, Fax: +492317555933.

Contract/grant sponsor: German Research Foundation (DFG); contract/grant number: TU102/34 
intensively been put forward in distinctive works such as in Ref. [9, 10, 11, 12] among others. Analytical solutions for one-dimensional (1-d) wave propagation in saturated poroelastic media are given in, e. g., Ref. [13, 14].

For the numerical treatment, saturated, materially incompressible porous media are represented by a set of differential-algebraic equations (DAE), which belong to the class of strongly coupled problems discussed, e.g., in Ref. [15, 16, 6, 17]. In particular, such multi-field problems cannot be solved in a straightforward manner as the governing set of DAE includes algebraic equations representing some essential side condition or Lagrangian constraint, for instance, forcing incompressibility or continuity. This typically leads to ill-conditioned saddle-point problems, which become even more vulnerable to stability and robustness issues if they are accompanied by a certain roughness of the model parameters associated with a tight coupling of the equations, cf. Ref. [17].

In this regard, a novel monolithic Newton-multigrid-based finite element method (FEM) solution approach has been recently proposed by the authors in Ref. [17] to solve the arising coupled multifield problem for the case of a $\mathbf{u v} p$ formulation (where the governing set of equations are the balance of momentum of the solid phase, the balance of momentum of the fluid phase and the total volume balance. Here, the primary variables are $\mathrm{u}, \mathrm{v}$ and $\mathrm{p}$ which refer to the field variables solid displacement, fluid velocity and pore-fluid pressure). This choice appeared to be more accurate, stable and efficient than previous methods in many test cases. However, this approach is restricted to infinitesimal deformations so that the porosity can be considered as constant parameter.

In the underlying research work, we introduce a new numerical time-stepping approach based on a new uw $p$ formulation deploying the mixture momentum balance, the fluid momentum balance and the total volume balance with $\mathrm{u}, \mathrm{w}, \mathrm{p}$ being the primary variables where $\mathrm{w}$ indicates the filter velocity. The new approach borrows some of its techniques from the previous one and further allows for solving general non-linear problems and enjoys several advantages against the preceding one.

Thus, the first step is to derive the weak or variational statements of the governing balance equations, and the second step is to apply a well-conceived finite element discretization, which meets the anticipated accuracy and stability requirements. Thereafter, a well-established and advanced fast solver of Newton-multigrid type that can handle systems with large condition numbers, thereby outperforming common single grid methods, is applied. In fact, having PDEs with elliptic character, due to the incompressibility, hierarchical multigrid techniques as pure solvers or as preconditioners in Krylov space solvers have proven their advantageous convergence behavior since they may lead to iteration numbers independent of the problem size. This property allows to design highly efficient solution schemes, particularly for large problem sizes.

To give an overview, Section 2 is concerned with the theoretical fundamentals of a macroscopic porous media modeling including a brief presentation of the TPM, the concept of volume fractions, the kinematics, the balance and constitutive modeling. To pave the way for the direct use of some special CFD techniques, Section 3 reformulates the governing set of the considered PDEs into Stokes-like form. In Section 4 , the numerical treatment of the coupled problem is described including the weak formulation, the spatial and temporal discretization as well as the final matrix system. The linear and non-linear solvers are then discussed in Section 5 . Section 6 is concerned with the numerical validation and evaluation of the proposed solution strategy for linear deformations by comparing the results of $1 \mathrm{D}$ and 2D benchmark simulations with published data. In Section 7, two analytical simulations are carried out to validate our approach for the case of nonlinearity, while in Section 8 the effects of the convection term and the volume fractions changes are quantitatively studied for the case of strong and weak coupling. In Section 9 , a new adaptive time stepping procedure is proposed to cope with rapid changes in the numbers of non-linear iterations. Finally, Section 10 gives a brief summary and conclusions of the presented research work.

\section{THEORETICAL FUNDAMENTALS}

The considered porous media bi-phase model consists of a deformable porous solid skeleton and a pore fluid. The solid material is assumed to be non-polar and incompressible, while the fluid material is assumed to be incompressible and viscous. The model is simplified by excluding thermal 
effects (isothermal model) as well as any mass exchanges between the constituents. Moreover, the permeability of such immiscible binary aggregates is assumed to be isotropic. As this paper concerns the numerical treatment of the related PDEs, only a brief physical description is introduced to define the terms of the examined PDEs. For a more detailed discussion, the interested reader is referred to [11, 12, 5, 18, 19] and the citations therein.

\subsection{Macroscopic Porous Media Approach}

In the following macroscopic approach, the binary aggregate is treated as a macroscopic mixture $\varphi$ with overlaid and interacting but de facto immiscible solid and fluid constituents $\varphi^{\alpha}(\alpha=S$ : solid; $\alpha=F$ : fluid), so that $\varphi=\varphi^{S} \cup \varphi^{F}$ at any macroscopic spatial point $\mathbf{x}(t)$ at any time $t \in\left[t_{0}, T\right]$. The local composition of the biphasic continuum is described by volume fractions $n^{\alpha}(\mathbf{x}, t):=\mathrm{d} v^{\alpha} / \mathrm{d} v \in[0,1]$ of $\varphi^{\alpha}\left(n^{S}:\right.$ solidity; $n^{F}:$ porosity) defined as the ratios of the partial to the total volume elements of $\varphi$. Assuming fully saturated conditions, the saturation constraint obviously yields

$$
\sum_{\alpha} n^{\alpha}=n^{S}+n^{F}=1
$$

Closely related is the introduction of two density functions, namely an effective density $\rho^{\alpha R}(\mathbf{x}, t)$ and a partial density $\rho^{\alpha}(\mathbf{x}, t)$ relating the local mass of $\varphi^{\alpha}$ to the partial or the bulk volume element, respectively. It is easily concluded that

$$
\rho^{\alpha}=n^{\alpha} \rho^{\alpha R}, \quad \alpha \in\{S, F\}
$$

revealing the general compressibility of porous solids through possible changes of the pore space. Moreover, $\rho=\sum_{\alpha} \rho^{\alpha}$ represents the mixture density.

Following this, the kinematics of multiphase materials within a continuum-mechanical treatment proceeds from superimposed and interacting continua but with individual unique states of motion for each constituent, i.e. the velocity and the acceleration fields, respectively, read

$$
\begin{aligned}
& \mathbf{v}_{\alpha}:=\mathbf{x}_{\alpha}^{\prime}, \quad\left(\mathbf{v}_{\alpha}\right)_{\alpha}^{\prime}=\stackrel{\mathbf{x}}{\alpha}_{\alpha}^{\prime \prime} \text { with } \\
& (\cdot)_{\alpha}^{\prime}:=\frac{\mathrm{d}_{\alpha}(\cdot)}{\mathrm{d} t}=\frac{\partial(\cdot)}{\partial t}+\operatorname{grad}(\cdot) \cdot \mathbf{v}_{\alpha},
\end{aligned}
$$

where the material time derivative of a certain quantity $(\bullet)$ follows the motion of $\varphi^{\alpha}$ and $\operatorname{grad}(\cdot):=\partial(\cdot) / \partial \mathbf{x}$. In porous media theories, it is convenient to proceed from a Lagrangian description of the solid matrix via the solid displacement $\mathbf{u}_{S}$ and velocity $\mathbf{v}_{S}$ as the kinematic variables. However, the pore-fluid flow is expressed either in a modified Eulerian setting via the seepage velocity vector $\mathbf{w}_{F S}$ describing the fluid motion relative to the deforming skeleton, or by an Eulerian description using the fluid velocity $\mathbf{v}_{F}$ itself. In this connection, the so-called filter velocity $\mathbf{w}$ can be introduced as an additional kinematic quantity relating $\mathbf{w}_{F S}$ to the fluid part of the mixture. In particular, we have

$$
\begin{aligned}
& \mathbf{u}_{S}=\mathbf{x}-\mathbf{X}_{S}, \quad \mathbf{v}_{S}=\left(\mathbf{u}_{S}\right)_{S}^{\prime}=\dot{x}_{S}^{\prime}, \\
& \mathbf{v}_{F}=\mathbf{x}_{F}^{\prime}, \quad \mathbf{w}_{F S}=\mathbf{v}_{F}-\mathbf{v}_{S}, \quad \mathbf{w}=n^{F} \mathbf{w}_{F S}
\end{aligned}
$$

with $\mathbf{X}_{S}$ representing the position vector of the solid phase in the reference configuration and $\mathbf{x}$ is the position vector in the current configuration.

\subsection{Governing Balance Relations}

\section{Volume Balance}

Each constituent must undergo the law of conservation of mass, which is given under the assumption of no mass exchange between the constituents as

$$
\left(\rho^{\alpha}\right)_{\alpha}^{\prime}+\rho^{\alpha} \operatorname{div}\left(\mathbf{v}_{\alpha}\right)=0, \quad \alpha \in\{S, F\},
$$


where $\operatorname{div}(\bullet)$ is the divergence operator related to $\operatorname{grad}(\bullet)$. Moreover, under the assumption of materially incompressible constituents

$$
\rho^{\alpha R}=\text { const. }, \quad \alpha \in\{S, F\},
$$

relation (5) reduces to the following volume balance equation:

$$
\left(n^{\alpha}\right)_{\alpha}^{\prime}+n^{\alpha} \operatorname{div}\left(\mathbf{v}_{\alpha}\right)=0, \quad \alpha \in\{S, F\} .
$$

The current volume fractions $n^{\alpha}$ can be computed with the help of the initial solid volume fraction $n_{0 S}^{\mathrm{S}}$ via

$$
n^{\mathrm{S}}=\operatorname{det}\left(\mathbf{F}_{S}^{-1}\right) n_{0 S}^{\mathrm{S}}=\frac{n_{0 S}^{S}}{J_{\mathrm{S}}} .
$$

Summation of the left hand sides of the two volume balance equations (7) for $\alpha \in\{\mathrm{F}, \mathrm{S}\}$ and then using (1) and (3) yields the continuity-like volume balance equation of the overall aggregate

$$
\operatorname{div}\left(n^{\mathrm{F}} \mathbf{v}_{\mathrm{F}}+n^{\mathrm{S}} \mathbf{v}_{\mathrm{S}}\right)=0 .
$$

\section{Momentum Balance}

The constituent momentum balance is given by

$$
\rho^{\alpha}\left(\mathbf{v}_{\alpha}\right)_{\alpha}^{\prime}=\operatorname{div} \mathbf{T}^{\alpha}+\rho^{\alpha} \mathbf{b}+\hat{\mathbf{p}}^{\alpha},
$$

where $\mathbf{T}^{\alpha}$ is the symmetric Cauchy stress tensor for the constituent $\varphi^{\alpha}, \mathbf{b}$ is the mass-specific body force uniformly acting on the overall aggregate, and $\hat{\mathbf{p}}^{\alpha}$ denotes the direct momentum production, which can be interpreted as the volume-specific local interaction force between the percolating pore fluid and the solid skeleton. Due to the overall conservation of momentum,

$$
\hat{\mathbf{p}}^{S}+\hat{\mathbf{p}}^{F}=\mathbf{0}
$$

must hold. Making use of the concept of effective stresses to describe the stress state in a material point of a homogenized porous material, the partial stress and the fluid interaction force are additively split as follows

$$
\begin{aligned}
\mathbf{T}^{\alpha} & =\mathbf{T}_{\mathrm{E}}^{\alpha}-n^{\alpha} p \mathbf{I}, \\
\hat{\mathbf{p}}^{\mathrm{F}} & =p \operatorname{grad}\left(n^{\mathrm{F}}\right)+\hat{\mathbf{p}}_{\mathrm{E}}^{\mathrm{F}} .
\end{aligned}
$$

Therein, $\mathbf{T}_{\mathrm{E}}^{\alpha}$ and $\hat{\mathbf{p}}_{\mathrm{E}}^{\mathrm{F}}$ are the so-called 'extra' or 'effective' terms and $p$ is the pore-fluid pressure, which can also be interpreted as Lagrangian multiplier for the incompressibility requirement. It follows from a dimensional analysis of the laminar flow in porous media on the micro and macro scales that $\operatorname{div}\left(\mathbf{T}_{\mathrm{E}}^{\mathrm{F}}\right) \ll \hat{\mathbf{p}}_{\mathrm{E}}^{\mathrm{F}}$ and thus the term $\operatorname{div}\left(\mathbf{T}_{\mathrm{E}}^{\mathrm{F}}\right)$ can be dropped from the fluid momentum balance, cf. [20]. In case of isotropic permeability, the interaction force can be written as

$$
\hat{\mathbf{p}}_{\mathrm{E}}^{\mathrm{F}}=-\frac{\left(n^{\mathrm{F}}\right)^{2} \gamma^{\mathrm{FR}}}{k^{\mathrm{F}}} \mathbf{w}_{F S},
$$

where $\gamma^{\mathrm{FR}}=\rho^{\mathrm{FR}}|\mathbf{b}|$ is the effective weight of the fluid, $|\mathbf{b}|=g=$ const. is the gravity and $k^{\mathrm{F}}$ the Darcy permeability parameter. The left hand side of the momentum balance equation (10) for the fluid constituent can then be rewritten as follows

$$
\begin{aligned}
& \operatorname{div}\left(\mathbf{T}^{\mathrm{F}}\right)+\rho^{\mathrm{F}} \mathbf{b}+\hat{\mathbf{p}}^{\mathrm{F}} \\
= & \underbrace{\operatorname{div}\left(\mathbf{T}_{\mathrm{E}}^{\mathrm{F}}\right)}_{\approx \mathbf{0}}-\operatorname{div}\left(n^{\mathrm{F}} p \mathbf{I}\right)+\rho^{\mathrm{F}} \mathbf{b}+p \operatorname{grad}\left(n^{\mathrm{F}}\right)+\hat{\mathbf{p}}_{\mathrm{E}}^{\mathrm{F}} \\
= & -n^{\mathrm{F}} \operatorname{grad}(p)-p \operatorname{grad}\left(n^{\mathrm{F}}\right)+\rho^{\mathrm{F}} \mathbf{b}+p \operatorname{grad}\left(n^{\mathrm{F}}\right)+\hat{\mathbf{p}}_{\mathrm{E}}^{\mathrm{F}} \\
= & -n^{\mathrm{F}} \operatorname{grad}(p)+\rho^{\mathrm{F}} \mathbf{b}-\frac{\left(n^{\mathrm{F}}\right)^{2} \gamma^{\mathrm{FR}}}{k^{\mathrm{F}}} \mathbf{w}_{F S} .
\end{aligned}
$$


Hence, the momentum balance equation (10) for the fluid constituent simplifies to

$$
\rho^{\mathrm{F}}\left(\mathbf{v}_{\mathrm{F}}\right)_{\mathrm{F}}^{\prime}+n^{\mathrm{F}} \operatorname{grad}(p)-\rho^{\mathrm{F}} \mathbf{b}+\frac{\left(n^{\mathrm{F}}\right)^{2} \gamma^{\mathrm{FR}}}{k^{\mathrm{F}}} \mathbf{w}_{F S}=\mathbf{0} .
$$

The Darcy permeability parameter $k^{\mathrm{F}}$, which relates the filter velocity $n^{\mathrm{F}} \mathbf{w}_{F S}$ to the pressure gradient $\operatorname{grad}(p)$, depends on the fluid's viscosity and the micro-topology of the pore space. In general, $k^{\mathrm{F}}$ is deformation dependent and can for instant be expressed as a function of $n^{\mathrm{F}}$ according to [19] as

$$
k^{\mathrm{F}}\left(n^{\mathrm{F}}\right)=k_{0}^{\mathrm{F}}\left(\frac{n^{\mathrm{F}}}{n_{0}^{\mathrm{F}}}\right)^{\kappa},
$$

where $k_{0}^{\mathrm{F}}$ is the initial permeability, $n_{0}^{\mathrm{F}}=1-n_{0 S}^{\mathrm{S}}$ is the initial fluid volume fraction, and the parameter $\kappa \geq 0$ determines the strength of the deformation dependency ( $\kappa=0 \Rightarrow k^{\mathrm{F}}=$ const.). For further particulars see also [1]. Moreover, using (3), the material time derivative of $\mathbf{v}_{\mathrm{F}}$ with respect to the fluid motion can be written as

$$
\left(\mathbf{v}_{\mathrm{F}}\right)_{\mathrm{F}}^{\prime}=\left(\mathbf{v}_{\mathrm{F}}\right)_{\mathrm{S}}^{\prime}+\operatorname{grad}\left(\mathbf{v}_{\mathrm{F}}\right) \mathbf{w}_{F S} .
$$

By use of (4) and (17), the momentum balance for the fluid constituent (15) reads

$$
\rho^{\mathrm{F}}\left(\mathbf{v}_{\mathrm{F}}\right)_{\mathrm{S}}^{\prime}+n^{\mathrm{F}} \operatorname{grad}(p)-\rho^{\mathrm{F}} \mathbf{b}+\rho^{\mathrm{F}}\left(\operatorname{grad}\left(\mathbf{v}_{\mathrm{F}}\right)+\frac{g n^{\mathrm{F}}}{k^{\mathrm{F}}} \mathbf{I}\right)\left(\mathbf{v}_{F}-\mathbf{v}_{S}\right)=\mathbf{0} .
$$

By use of (10), (11), (13) and (14), the momentum balance for solid constituent reads

$$
\rho^{\mathrm{S}}\left(\mathbf{v}_{\mathbf{S}}\right)_{\mathrm{S}}^{\prime}-\operatorname{div}\left(\mathbf{T}_{E}^{S}\right)+n^{\mathrm{S}} \operatorname{grad}(p)-\rho^{\mathrm{S}} \mathbf{b}-\frac{\left(n^{\mathrm{F}}\right)^{2} \gamma^{\mathrm{FR}}}{k^{\mathrm{F}}}\left(\mathbf{v}_{F}-\mathbf{v}_{S}\right)=\mathbf{0},
$$

where $\mathbf{T}_{E}^{S}$ is the solid Cauchy extra stress tensor will be explained in Section 2.3 . In summary, the PDEs we seek to solve read:

- Balance of momentum of the solid phase:

$$
\rho^{S}\left(\mathbf{v}_{S}\right)_{S}^{\prime}=\operatorname{div}\left(\mathbf{T}_{E}^{S}\right)+\rho^{S} \mathbf{b}+\frac{\left(n^{F}\right)^{2} \gamma^{F R}}{k^{F}}\left(\mathbf{v}_{F}-\mathbf{v}_{S}\right)-n^{S} \operatorname{grad}(p),
$$

- Balance of momentum of the fluid phase:

$$
\rho^{F}\left(\mathbf{v}_{F}\right)_{S}^{\prime}+\rho^{\mathrm{F}} \operatorname{grad}\left(\mathbf{v}_{\mathrm{F}}\right)\left(\mathbf{v}_{F}-\mathbf{v}_{S}\right)=\rho^{F} \mathbf{b}-\frac{\left(n^{F}\right)^{2} \gamma^{F R}}{k^{F}}\left(\mathbf{v}_{F}-\mathbf{v}_{S}\right)-n^{F} \operatorname{grad}(p),
$$

- Volume balance of the overall aggregate:

$$
\operatorname{div}\left(n^{F} \mathbf{v}_{F}\right)+\operatorname{div}\left(n^{S} \mathbf{v}_{S}\right)=0 .
$$

Note that the chosen primary unknowns for this set of PDE are $\mathbf{u}_{S}, \mathbf{v}_{F}$ and $p$. Hence, $\mathbf{v}_{S}\left(\mathbf{u}_{S}\right)$ as well as $\mathbf{T}_{E}^{S}\left(\mathbf{u}_{S}\right), n^{S}\left(\mathbf{u}_{S}\right), n^{F}\left(\mathbf{u}_{S}\right)$ and $\mathbf{w}_{F S}$ represent the secondary variables of the problem. Additionally, a reduction in the order of the PDE to order one in time is achieved using

$$
\left(\mathbf{u}_{S}\right)_{S}^{\prime}=\mathbf{v}_{S},
$$

which eliminates the second time derivative of the solid displacement from (20), and allows the applicability of a wide range of fundamental time-stepping algorithms. Finally, equations (20)-(23) are referred to as $\mathbf{u v} p$ formulation. 


\subsection{Constitutive Relations of the Solid Porous Medium}

For infinitesimal linear deformations, the solid extra stress $\mathbf{T}_{E}^{S}$ is determined by the Hookean elasticity law

$$
\mathbf{T}_{E}^{S}=2 \mu^{S} \boldsymbol{\varepsilon}_{S}+\lambda^{S}\left(\boldsymbol{\varepsilon}_{S} \cdot \mathbf{I}\right) \mathbf{I} \quad \text { with } \quad \boldsymbol{\varepsilon}_{S}=\frac{1}{2}\left[\operatorname{grad}\left(\mathbf{u}_{S}\right)+\operatorname{grad}^{T}\left(\mathbf{u}_{S}\right)\right] .
$$

In this case, $n^{S} \approx n_{0 S}^{S}$ and $k^{F}=k_{0 S}^{F}$. For non-linear finite deformations, the material response is described using a Neo-Hookean-type hyper-elastic constitutive model. The corresponding strain energy function can be expressed as

$$
W^{\mathrm{S}}=\frac{1}{2} \mu^{S}\left[\operatorname{tr}\left(\mathbf{C}_{\mathrm{S}}\right)-3\right]-\mu^{S} \ln \left(J_{\mathrm{S}}\right)+U^{\mathrm{S}}\left(J_{\mathrm{S}}\right),
$$

where $\mathbf{C}_{S}=\mathbf{F}_{S}^{\mathrm{T}} \mathbf{F}_{S}$ is the right Cauchy-Green deformation tensor, $\mu^{S}$ is the macroscopic shear modulus (first Lamé parameter) of the solid matrix and $U^{\mathrm{S}}\left(J_{\mathrm{S}}\right)$ is a function describing the response to volumetric deformations. Although the solid skeleton is materially incompressible, the solid matrix is compressible; large volume variations are possible due to the changes of the solid volume fraction. Based on the relation between the solid volume fraction $n^{\mathrm{S}}$ and the determinant of the solid deformation gradient $J_{\mathrm{S}}=\operatorname{det}\left(\mathbf{F}_{S}\right)$ (see equation (8)) and due to the constraint $n^{\mathrm{S}}<1$, the estimate

$$
n_{0 S}^{\mathrm{S}}<J_{\mathrm{S}}<\infty
$$

holds, i.e., the initial solid volume fraction is a lower bound for the determinant of the solid deformation gradient. In this connection, Eipper [19] proposed the following thermodynamicallyconsistent constitutive law that complies to this constraint

$$
U^{\mathrm{S}}\left(J_{\mathrm{S}}\right)=\lambda^{S}\left(1-n_{0 S}^{\mathrm{S}}\right)^{2}\left[\frac{J_{\mathrm{S}}-1}{1-n_{0 S}^{\mathrm{S}}}-\ln \left(\frac{J_{\mathrm{S}}-n_{0 S}^{\mathrm{S}}}{1-n_{0 S}^{\mathrm{S}}}\right)\right] .
$$

Here, $\lambda^{S}$ is the second Lamé parameter. The solid Cauchy extra stress tensor $\mathbf{T}_{\mathrm{E}}^{\mathrm{S}}=\frac{1}{J_{\mathrm{S}}} \mathbf{F}_{S}\left(2 \frac{\partial W^{\mathrm{S}}}{\partial \mathbf{C}_{\mathrm{S}}}\right) \mathbf{F}_{S}^{\mathrm{T}}$ corresponding to equations (25) and (27) is then given by

$$
\mathbf{T}_{\mathrm{E}}^{\mathrm{S}}=\frac{\mu^{S}}{J_{\mathrm{S}}}\left(\mathbf{F}_{S} \mathbf{F}_{S}^{\mathrm{T}}-\mathbf{I}\right)+\lambda^{S}\left(1-n_{0 S}^{\mathrm{S}}\right)^{2}\left(\frac{1}{1-n_{0 S}^{\mathrm{S}}}-\frac{1}{J_{\mathrm{S}}-n_{0 S}^{\mathrm{S}}}\right) \mathbf{I} .
$$

Alternative formulations and further discussions on the strain energy and its volumetric extension can be found in [2].

\section{NEW uw $p$ FORMULATION}

The aim in the following is to prepare for application of some special CFD techniques, originally designed to solve the incompressible Stokes and Navier-Stokes equations, to treat the non-linear system of PDEs (20)-(23). To apply these techniques (already discussed for the case of infinitesimal deformation in [17]), the PDEs need to be written in the Stokes-like or Navier-Stokes-like form to exploit the formal similarities of the model equations of CFD, i. e., our system of equations must be similar to the following form

$$
\left(\begin{array}{cc}
\mathbf{C} & \mathbf{D} \\
\mathbf{D}^{\mathrm{T}} & \mathbf{0}
\end{array}\right)\left(\begin{array}{l}
\mathbf{v} \\
p
\end{array}\right)=\left(\begin{array}{c}
\mathbf{f}(\mathbf{v}) \\
\mathbf{0}
\end{array}\right)
$$

with $\mathbf{D}$ representing the gradient operator acting on the pressure $p, \mathbf{D}^{\mathrm{T}}$ the adjoint divergence operator, $\mathbf{C}$ a differential operator ${ }^{\dagger}$ acting on the fluid velocity vector $\mathbf{v}$ and $\mathbf{f}(\mathbf{v})$ is the right hand

${ }^{\dagger}$ For stationary Stokes problem, for instance, this is related to the laplace operator. 
side source function. To achieve this, a series of derivations and reformulations should be carried out.

To allow for the direct use of the standard $\theta$-scheme, we start by differentiating $\left(\rho \mathbf{v}_{S}\right)$ by parts which gives the following useful relation

$$
\left(\rho \mathbf{v}_{S}\right)_{S}^{\prime}=(\rho)_{S}^{\prime} \mathbf{v}_{S}+\rho\left(\mathbf{v}_{S}\right)_{S}^{\prime} \rightarrow \rho\left(\mathbf{v}_{S}\right)_{S}^{\prime}=\left(\rho \mathbf{v}_{S}\right)_{S}^{\prime}-(\rho)_{S}^{\prime} \mathbf{v}_{S}
$$

with the mixture density

$$
\rho=\rho^{S}+\rho^{F} .
$$

For the same reason $\mathrm{w} / n^{F}$ is also differentiated by parts to obtain another useful relation

$$
\left(\frac{\mathbf{w}}{n^{F}}\right)_{S}^{\prime}=\frac{n^{F}(\mathbf{w})_{S}^{\prime}-\left(n^{F}\right)_{S}^{\prime} \mathbf{w}}{\left(n^{F}\right)^{2}} \rightarrow \rho^{F}\left(\frac{\mathbf{w}}{n^{F}}\right)_{S}^{\prime}=-\rho^{F R} \frac{\left(n^{F}\right)_{S}^{\prime}}{n^{F}} \mathbf{w}+\rho^{F R}(\mathbf{w})_{S}^{\prime} .
$$

Here $\left(n^{F}\right)_{S}^{\prime}$ is computed by virtue of (7) and condition (1) as

$$
\left(n^{F}\right)_{S}^{\prime}=\left(1-n^{S}\right)_{S}^{\prime}=-\left(n^{S}\right)_{S}^{\prime}=n^{S} \operatorname{div}\left(\mathbf{v}_{S}\right) .
$$

Multiplying both sides of (32) by $\rho^{F R}$ and using (2), we obtain

$$
\left(\rho^{F}\right)_{S}^{\prime}=\left(\rho^{F R} n^{F}\right)_{S}^{\prime}=n^{S} \rho^{F R} \operatorname{div}\left(\mathbf{v}_{S}\right),
$$

which can be exploited together with (30) as well as (5), (2) and (6) (with $\alpha=S$ ) to compute $(\rho)_{S}^{\prime}$ as

$$
(\rho)_{S}^{\prime}=\left(\rho^{S}+\rho^{F}\right)_{S}^{\prime}=-\left(\rho^{S R}-\rho^{F R}\right) n^{S} \operatorname{div}\left(\mathbf{v}_{S}\right) .
$$

Next, using (1) and (30), we add up (20) and (21) resulting in the mixture momentum balance, which has a positive effect on the performance of our multigrid solver as will be shown later:

$$
\rho^{S}\left(\mathbf{v}_{S}\right)_{S}^{\prime}+\rho^{F}\left(\mathbf{v}_{F}\right)_{S}^{\prime}+\rho^{F} \operatorname{grad} \mathbf{v}_{F}\left(\mathbf{v}_{F}-\mathbf{v}_{S}\right)-\operatorname{div} \mathbf{T}_{E}^{S}-\rho \mathbf{b}+\operatorname{grad} p=\mathbf{0} .
$$

The main purpose of this step is to remove the variable $n^{S}$ in front of $\operatorname{grad} p$ in (20) in order to get a Stokes-like form and to avoid arising of $\operatorname{grad} n^{S}$ (which contains second-order derivatives) in the weak form to allow for testing with lower order finite elements for the no-convection assumption. The appearance of $\operatorname{grad} n^{S}$ cannot be avoided if (20) is applied because the intention is to use discontinuous pressure finite elements that do not carry derivatives and hence the indispensable integration by parts (to remove the differential operator grad, acting on $p$ ) will generate the undesirable $\operatorname{grad} n^{S}$. Furthermore, this step, as will be shown later, facilitates the application of the boundary conditions.

For the same main purpose, (21) is divided by $n^{F}>0$ yielding

$$
\rho^{F R}\left(\mathbf{v}_{F}\right)_{S}^{\prime}+\rho^{F R} \operatorname{grad} \mathbf{v}_{F}\left(\mathbf{v}_{F}-\mathbf{v}_{S}\right)-\rho^{F R} \mathbf{b}+\frac{n^{F} \gamma^{F R}}{k^{F}}\left(\mathbf{v}_{F}-\mathbf{v}_{S}\right)+\operatorname{grad} p=\mathbf{0},
$$

which removes the leading coefficient $n^{F}$ before $\operatorname{grad} p$ and leads to a solution-independent external load vector $\left(\mathbf{t}^{F}\right)$, which depends only on the ambient pressure as will be shown in the next section. To continue with this reformulation to get a Stokes-like form, (22) should also be modified by use of the filter velocity vector $\mathrm{w}$

$$
\mathbf{v}_{F}=\mathbf{w} / n^{F}+\mathbf{v}_{S}, \quad \text { where } n^{\mathrm{F}}>0
$$

and then by substitution of (37) in (35), (36) and (22) and then making use of (29) and (31), we get the new $\mathbf{u w} p$ formulation: 
- Balance of momentum of the binary saturated mixture:

$$
\begin{aligned}
\left(\rho \mathbf{v}_{S}\right)_{S}^{\prime}+\rho^{F R}(\mathbf{w})_{S}^{\prime} & +\rho^{F R} \operatorname{grad} \mathbf{v}_{F} \mathbf{w}-\operatorname{div} \mathbf{T}_{E}^{S}-\rho \mathbf{b} \\
& -(\rho)_{S}^{\prime} \mathbf{v}_{S}-\frac{\left(\rho^{F}\right)_{S}^{\prime}}{n^{F}} \mathbf{w}+\operatorname{grad} p=\mathbf{0},
\end{aligned}
$$

- Balance of momentum of the fluid phase:

$$
\begin{aligned}
\rho^{F R}\left(\mathbf{v}_{S}\right)_{S}^{\prime}+\rho^{F R}\left(\frac{\mathbf{w}}{n^{F}}\right)_{S}^{\prime} & +\frac{\rho^{F R}}{n^{F}} \operatorname{grad} \mathbf{v}_{F} \mathbf{w} \\
& +\frac{\gamma^{F R}}{k^{F}} \mathbf{w}-\rho^{F R} \mathbf{b}+\operatorname{grad} p=\mathbf{0},
\end{aligned}
$$

- Volume balance of the binary saturated mixture:

$$
\operatorname{div}\left(\mathbf{v}_{S}\right)+\operatorname{div}(\mathbf{w})=0
$$

- Velocity-displacement relationship:

$$
\left(\mathbf{u}_{S}\right)_{S}^{\prime}=\mathbf{v}_{S}
$$

The convective terms and the volume fraction changes are colored with orange and green, respectively, because we will refer to them frequently when later studying their influence. Note that the chosen primary unknowns for this set of PDE are $\mathbf{u}_{S}, \mathbf{w}$ and $p$. Hence, $\mathbf{v}_{S}\left(\mathbf{u}_{S}\right)$ as well as $\mathbf{T}_{E}^{S}\left(\mathbf{u}_{S}\right), n^{S}\left(\mathbf{u}_{S}\right), n^{F}\left(\mathbf{u}_{S}\right)$ and $\mathbf{v}_{F}$ represent the secondary variables of the problem. The spatial discretization of (38)-(41) is applied using the Finite Element Method, whereas the time integration can be carried out using the $\theta$-scheme as described in [17] in which $\nabla p$, (40) and (41) are always treated fully implicitly with $\theta=1$ as will be explained in the following.

\section{WEAK FORMULATION AND DISCRETIZATION IN SPACE AND TIME}

Our subsequent variational form of the uw $p$ approach is created by multiplying (38), (39), (40) and (41) with the displacement test function $\delta \mathbf{u}_{S}$, the filter velocity test function $\delta \mathbf{w}$, the pressure test function $\delta p$ and the velocity test function $\delta \mathbf{v}_{S}$, respectively, integrating over the current domain $\Omega(t)$ and performing partial integrations. Finally, we obtain the following weak form:

$$
\begin{aligned}
& \underbrace{\int_{\Omega(t)} \operatorname{grad} \delta \mathbf{u}_{S}: \mathbf{T}_{E}^{S} \mathrm{~d} v^{t}}_{\mathbf{K}_{\mathbf{u u}}+\mathbf{h} \text { see appendix }} \overbrace{-\int_{\Omega(t)} p \operatorname{div} \delta \mathbf{u}_{S} \mathrm{~d} v^{t}}^{\mathbf{K}_{\mathbf{u} p}}+\underbrace{\int_{\Omega(t)} \delta \mathbf{u}_{S} \cdot\left(\rho \mathbf{v}_{S}\right)_{S}^{\prime} \mathrm{d} v^{t}}_{\mathbf{M}_{\mathbf{u v}}} \\
& +\underbrace{\rho^{F R} \int_{\Omega(t)} \delta \mathbf{u}_{S} \cdot(\mathbf{w})_{S}^{\prime} \mathrm{d} v^{t}}_{\mathbf{M}_{\mathbf{u w}}} \underbrace{+\rho^{F R} \int_{\Omega(t)} \delta \mathbf{u}_{S} \cdot\left(\operatorname{grad}\left(\mathbf{v}_{F}\right)-\frac{\left(n^{F}\right)_{S}^{\prime}}{n^{F}} \mathbf{I}\right) \mathbf{w} \mathrm{d} v^{t}}_{\mathbf{K}_{\mathbf{w}}} \\
& \underbrace{-\int_{\Omega(t)} \delta \mathbf{u}_{S} \cdot(\rho)_{S}^{\prime} \mathbf{v}_{S} \mathrm{~d} v^{t}}_{\mathbf{K}_{\mathbf{u v}}}=\underbrace{\int_{\Omega(t)} \rho \delta \mathbf{u}_{S} \cdot \mathbf{b} \mathrm{d} v+\int_{\Gamma_{\mathbf{t}}}^{\int_{\mathbf{u w}}} \delta \mathbf{u}_{S} \cdot \overline{\mathbf{t}} \mathrm{d} a^{t}}_{\mathbf{f}_{m}},
\end{aligned}
$$




$$
\begin{aligned}
& \underbrace{\rho^{F R} \int_{\Omega(t)} \delta \mathbf{w} \cdot\left(\frac{1}{n^{F}} \operatorname{grad}\left(\mathbf{v}_{F}\right)+\frac{g}{k^{F}} \mathbf{I}\right) \mathbf{w} \mathrm{d} v^{t}}_{\mathbf{K}_{\mathbf{w w}}}-\overbrace{\int_{\Omega(t)} p \operatorname{div} \delta \mathbf{w} \mathrm{d} v^{t}}^{\mathbf{K}_{\mathbf{w} p}} \\
& +\underbrace{\rho^{F R} \int_{\Omega(t)} \delta \mathbf{w} \cdot\left(\frac{\mathbf{w}}{n^{F}}\right)_{S}^{\prime} \mathrm{d} v^{t}}_{\mathbf{M}_{\mathbf{w w}}}+\underbrace{\rho^{F R} \int_{\Omega(t)} \delta \mathbf{w} \cdot\left(\mathbf{v}_{S}\right)_{S}^{\prime} \mathrm{d} v^{t}}_{\mathbf{M}_{\mathbf{w v}}} \\
& =\underbrace{\rho^{F R} \int_{\Omega(t)} \delta \mathbf{w} \cdot \mathbf{b} \mathrm{d} v^{t}+\int_{\Gamma_{\mathbf{t}} F} \delta \mathbf{w} \cdot \overline{\mathbf{t}}^{F} \mathrm{~d} a^{t}}_{\mathbf{f}_{\mathbf{w}}}, \\
& \overbrace{\int_{\Omega(t)} \delta p \operatorname{div} \mathbf{v}_{S} \mathrm{~d} v^{t}}^{\mathbf{K}_{p \mathbf{v}}}+\overbrace{\int_{\Omega(t)} \delta p \operatorname{div} \mathbf{w} \mathrm{d} v^{t}}^{\mathbf{K}_{p \mathbf{w}}}=0, \\
& \underbrace{\int_{\Omega(t)} \delta \mathbf{v}_{S} \cdot\left(\mathbf{u}_{S}\right)_{S}^{\prime} \mathrm{d} v^{t}}_{\mathbf{M}_{\mathbf{v u}}} \underbrace{-\int_{\Omega(t)} \delta \mathbf{v}_{S} \cdot \mathbf{v}_{S} \mathrm{~d} v^{t}}_{\mathbf{K}_{\mathbf{v v}}}=0 .
\end{aligned}
$$

The boundary $\Gamma=\partial \Omega$ is divided into Dirichlet (essential) and Neumann (natural) boundaries, respectively, resulting in $\Gamma=\Gamma_{\mathbf{u}_{S}} \cup \Gamma_{\mathbf{t}}$ for the mixture balance of momentum and in $\Gamma=\Gamma_{\mathbf{w}} \cup \Gamma_{\mathbf{t}^{F}}$ for the balance of momentum of the fluid phase, wherein the corresponding tractions are defined, respectively, as

$$
\overline{\mathbf{t}}=\left[\mathbf{T}_{E}^{S}-p \mathbf{I}\right] \mathbf{n}, \quad \overline{\mathbf{t}}^{F}=-p \mathbf{n}
$$

Based on [5] and [17], the above boundary conditions are now more convenient to impose, not only because one can appoint them independently (due to the absence of the volume efflux as additional boundary condition) but also $\overline{\mathbf{t}}^{F}$ (as being free of $n^{F}$ ) depends only on the ambient pressure while the surface traction $\overline{\mathbf{t}}$ acts simultaneously on both the solid and the fluid phase such that the segregation of the boundary conditions is dispensable

So far, we have only discretized in space. The purpose was to show the Neumann boundary conditions (46) and to label the bilinear forms $\left(\mathbf{K}_{\mathbf{u u}}, \mathbf{K}_{\mathbf{u} p} \ldots\right.$ etc). To have full discretization (in space and time), we further apply the theta scheme, described in [17], on (42)-(45), which leads eventually to the following task, where

$$
\tilde{\mathbf{u}}=\left[\begin{array}{c}
\mathbf{u}_{S} \\
\mathbf{v}_{S} \\
\mathbf{w}
\end{array}\right] \quad \text { and } \quad \tilde{p}=p \Delta t \quad \text { and } \quad \mathbf{y}=\left[\begin{array}{c}
\tilde{\mathbf{u}} \\
\tilde{p}
\end{array}\right]
$$

Given $\mathbf{y}^{n}$ and the time step $\Delta t=t_{n+1}-t_{n}$, then solve for $\mathbf{y}=\mathbf{y}^{n+1}$

$$
\mathbf{A}(\mathbf{y}) \mathbf{y}=\mathbf{g}(\mathbf{y})
$$

with right hand side

$$
\mathbf{g}(\mathbf{y})=\mathbf{r}+\theta \Delta t \mathbf{f}(\mathbf{y})
$$


where $\Delta t=t_{n+1}-t_{n}$ denotes the step size, $t_{n}$ is the current time step, $t_{n+1}$ is the next time step and $(.)^{n}$ and $(.)^{n+1}$ are quantities in the current and the next time step, respectively. Furthermore,

$$
\begin{aligned}
& \mathbf{A}(\mathbf{y})=\left(\begin{array}{cccc}
\mathbf{M}_{\mathbf{v u}} & \theta_{1} \mathbf{K}_{\mathbf{v v}} & \mathbf{0} & \mathbf{0} \\
\theta_{1} \mathbf{K}_{\mathbf{u u}}\left(\mathbf{u}_{S}\right) & \mathbf{M}_{\mathbf{u v}}\left(\mathbf{u}_{S}\right)+\theta_{1} \mathbf{K}_{\mathbf{u v}}\left(\mathbf{u}_{S}, \mathbf{v}_{S}\right) & \mathbf{M}_{\mathbf{u w}}+\theta_{1} \mathbf{K}_{\mathbf{u w}}(\tilde{\mathbf{u}}) & \mathbf{K}_{\mathbf{u} p} \\
\mathbf{0} & \mathbf{M}_{\mathbf{w v}} & \mathbf{M}_{\mathbf{w w}}\left(\mathbf{u}_{S}\right)+\theta_{1} \mathbf{K}_{\mathbf{w w}}(\tilde{\mathbf{u}}) & \mathbf{K}_{\mathbf{w} p} \\
\mathbf{0} & \mathbf{K}_{p \mathbf{v}} & \mathbf{K}_{p \mathbf{w}} & \mathbf{0}
\end{array}\right) \\
& \mathbf{r}=\left(\begin{array}{cccc}
\mathbf{M}_{\mathbf{v u}} & \theta_{2} \mathbf{K}_{\mathbf{v v}} & \mathbf{0} & \mathbf{0} \\
\theta_{2} \mathbf{K}_{\mathbf{u u}}\left(\mathbf{u}_{S}^{n}\right) & \mathbf{M}_{\mathbf{u v}}\left(\mathbf{u}_{S}^{n}\right)+\theta_{2} \mathbf{K}_{\mathbf{u v}}\left(\mathbf{u}_{S}^{n}, \mathbf{v}_{S}^{n}\right) & \mathbf{M}_{\mathbf{u w}}+\theta_{2} \mathbf{K}_{\mathbf{u w}}\left(\tilde{\mathbf{u}}^{n}\right) & \mathbf{0} \\
\mathbf{0} & \mathbf{M}_{\mathbf{w v}} & \mathbf{M}_{\mathbf{w w}}\left(\mathbf{u}_{S}^{n}\right)+\theta_{2} \mathbf{K}_{\mathbf{w w}}\left(\tilde{\mathbf{u}}^{n}\right) & \mathbf{0} \\
\mathbf{0} & \mathbf{0} & \mathbf{0} & \mathbf{0}
\end{array}\right) \\
& \times\left(\begin{array}{c}
\mathbf{u}_{S}^{n} \\
\mathbf{v}_{S}^{n} \\
\mathbf{w}^{n} \\
\tilde{p}
\end{array}\right)+\theta_{2}\left(\begin{array}{c}
\mathbf{0} \\
\mathbf{f}_{\mathbf{u}}\left(\mathbf{u}_{S}^{n}\right) \\
\mathbf{f}_{\mathbf{w}}\left(\mathbf{u}_{S}^{n}\right) \\
\mathbf{0}
\end{array}\right),
\end{aligned}
$$

$$
\mathbf{g}(\mathbf{y})=\mathbf{r}+\theta \Delta t \underbrace{\left(\begin{array}{c}
\mathbf{0} \\
\mathbf{f}_{\mathbf{u}}\left(\mathbf{u}_{S}\right) \\
\mathbf{f}_{\mathbf{w}}\left(\mathbf{u}_{S}^{n}\right) \\
\mathbf{0}
\end{array}\right)}_{\mathbf{f}(\mathbf{y})}
$$

with

$$
\theta_{1}=\theta \Delta t, \quad \theta_{2}=(\theta-1) \Delta t .
$$

Equations (49) - (52) lead to Stokes-like saddle point problems

$$
\underbrace{\left(\begin{array}{cc}
\tilde{\mathbf{A}} & \mathbf{B} \\
\mathbf{B}^{\mathrm{T}} & \mathbf{0}
\end{array}\right)}_{\mathbf{A}} \underbrace{\left(\begin{array}{c}
\tilde{\mathbf{u}} \\
\tilde{p}
\end{array}\right)}_{\mathbf{y}}=\underbrace{\left(\begin{array}{c}
\mathbf{r h s} \tilde{\mathbf{u}} \\
\mathbf{0}
\end{array}\right)}_{\mathbf{g}(\mathbf{y})}
$$


with

$$
\tilde{\mathbf{A}}(\tilde{\mathbf{u}})=\left(\begin{array}{ccc}
\mathbf{M}_{\mathbf{v u}} & \theta_{1} \mathbf{K}_{\mathbf{v v}} & \mathbf{0} \\
\theta_{1} \mathbf{K}_{\mathbf{u u}}\left(\mathbf{u}_{S}\right) & \mathbf{M}_{\mathbf{u v}}\left(\mathbf{u}_{S}\right)+\theta_{1} \mathbf{K}_{\mathbf{u v}}\left(\mathbf{u}_{S}, \mathbf{v}_{S}\right) & \mathbf{M}_{\mathbf{u w}}+\theta_{1} \mathbf{K}_{\mathbf{u w}}(\tilde{\mathbf{u}}) \\
\mathbf{0} & \mathbf{M}_{\mathbf{w v}} & \mathbf{M}_{\mathbf{w w}}\left(\mathbf{u}_{S}\right)+\theta_{1} \mathbf{K}_{\mathbf{w w}}(\tilde{\mathbf{u}})
\end{array}\right)
$$

and

$$
\mathbf{B}=\left(\begin{array}{c}
\mathbf{0} \\
\mathbf{K}_{\mathbf{u} p} \\
\mathbf{K}_{\mathbf{w} p}
\end{array}\right)
$$

Therefore, we can directly use the special CFD techniques as presented in [17].

\section{ULP NON-LINEAR SOLVER}

To solve (54), we shall combine the updated Lagrangian approach (used in non-linear structural mechanics) with the pure Picard iteration method (often used in CFD) and we shall refer to this special non-linear solver as Updated Lagrangian-Picard solver or shortly ULP solver. In this algorithm, we only do operator evaluation with no additional Gateaux derivatives. Thus, the full Jacobian matrix is not used; in particular, the expensive material tangent matrix $\frac{\mathrm{d} \mathbf{T}_{E}^{S}}{\mathrm{~d} \mathbf{u}_{S}}$ is excluded here. For more details, see Algorithm 1

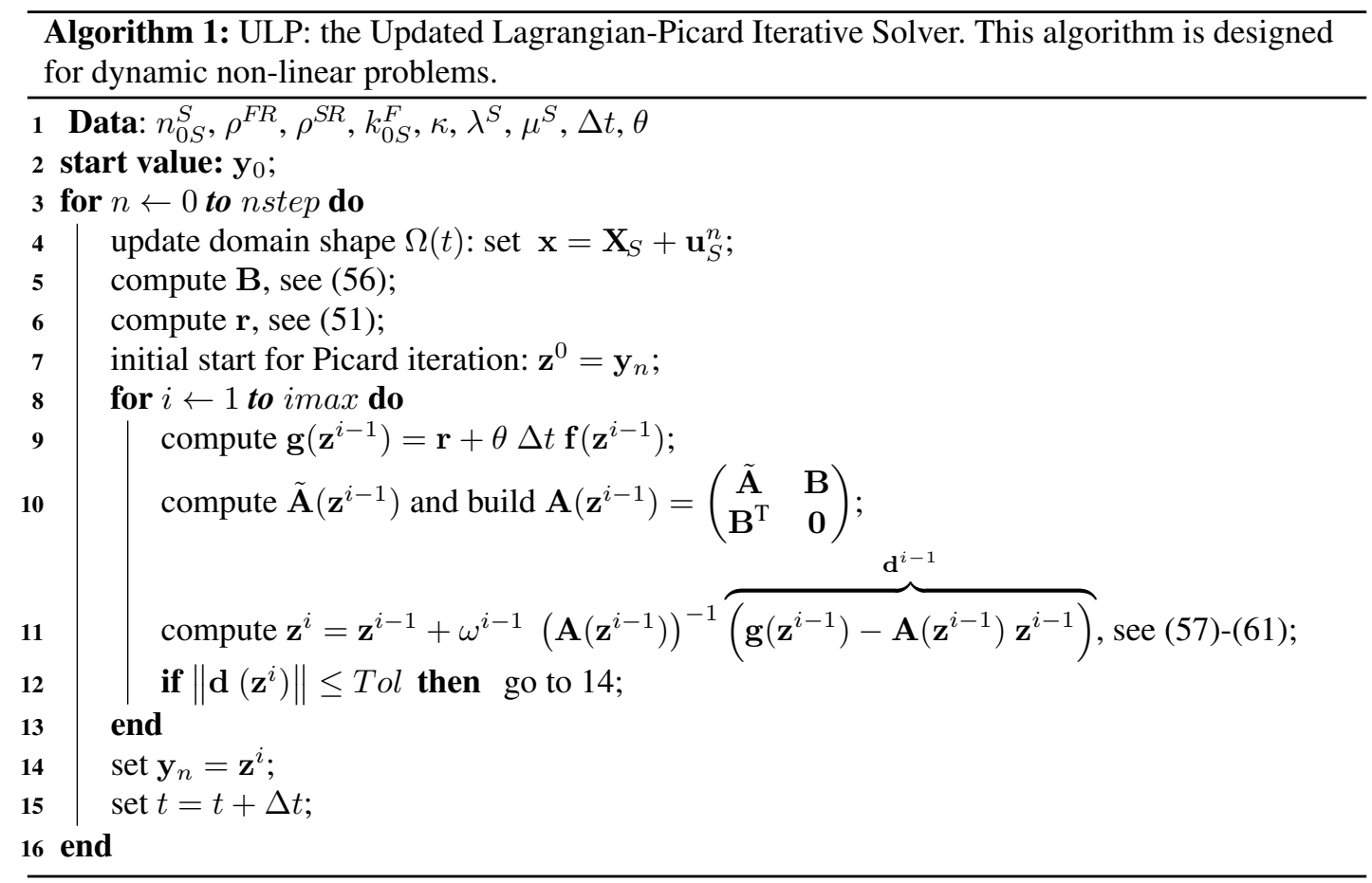

With regard to step 11 of this algorithm, 


$$
\mathbf{z}^{i}=\mathbf{z}^{i-1}+\omega^{i-1}\left(\mathbf{A}\left(\mathbf{z}^{i-1}\right)\right)^{-1} \overbrace{\left(\mathbf{g}\left(\mathbf{z}^{i-1}\right)-\mathbf{A}\left(\mathbf{z}^{i-1}\right) \mathbf{z}^{i-1}\right)}^{=\mathbf{d}^{i-1}},
$$

we follow the standard CFD procedure described in [21] in which (57) is split into the following three steps:

- Calculate the non-linear residual $\mathbf{d}^{i-1}$ :

$$
\mathbf{d}^{i-1}=\mathbf{g}\left(\mathbf{z}^{i-1}\right)-\mathbf{A}\left(\mathbf{z}^{i-1}\right) \mathbf{z}^{i-1},
$$

- Compute $\left(\mathbf{A}\left(\mathbf{z}^{i-1}\right)\right)^{-1} \mathbf{d}^{i-1}$ via iteratively or directly solving for $\mathbf{q}^{i-1}$

$$
\mathbf{A}\left(\mathbf{z}^{i-1}\right) \mathbf{q}^{i-1}=\mathbf{d}^{i-1}
$$

- Perform the updating step:

$$
\mathbf{z}^{i}=\mathbf{z}^{i-1}+\omega^{i-1} \mathbf{q}^{i-1}
$$

where the damping parameter $\omega^{i-1}$ in our case is set to 1 .

The iterations will continue unless the maximum number of iterations (imax) is reached or the norm of the non-linear residual goes below a given tolerance:

$$
\left\|\mathbf{d}^{i}\right\| \leq T o l .
$$

We use the special multigrid solver discussed in [17] (for more references see for example [22, 23, 24, 25], to solve (59) iteratively or UMFPACK to solve it directly.

It remains to mention that in the case of infinitesimal linear elastic deformations, updating the domain shape has almost no influence. Therefore, step 4 in Algorithm 1 is canceled. Hence, we end with the pure PICard solver) described in Algorithm 2 .

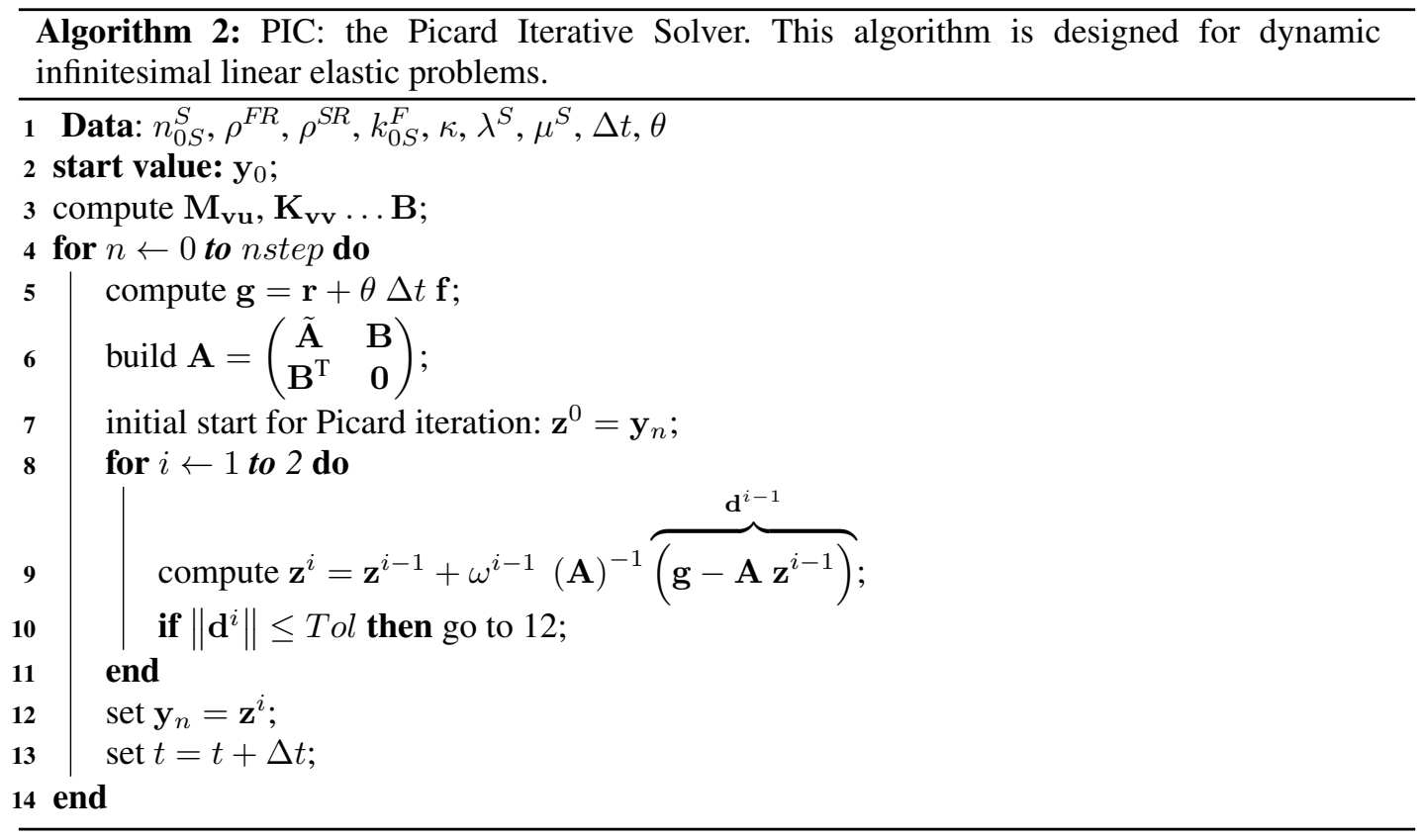




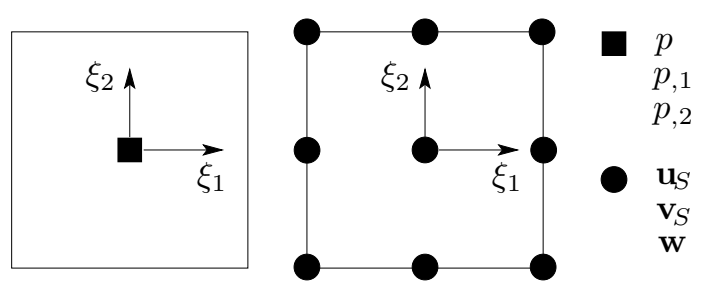

Figure 1. The discontinuous linear pressure element P1 (left) and the 9-node Lagrange bi-quadratic element Q2 (right) that are used for the uw $p$-TR method and $\xi_{1}$ and $\xi_{2}$ are the local element coordinates.

Table I. Averaged number of iterations (\# Iter.) and elapsed CPU time (CPU) in seconds per time step for the described multigrid solver for $\operatorname{uv} p(3)-\mathrm{TR}-\mathrm{Q} 2 / \mathrm{P} 1$ and $\mathbf{u w} p$-TR-Q2/P1 for the first 10 time steps and for the unstructured grid and $k_{0 S}^{F}=10^{-10}[\mathrm{~m} / \mathrm{s}]$. Here, Div. indicates divergence of the solver.

\begin{tabular}{|c|c|c|c|c|c|c|c|c|c|c|c|c|}
\hline \multicolumn{13}{|c|}{ time step size $=50 \times 10^{-3}[\mathrm{~s}]$} \\
\hline \multirow{3}{*}{ Level } & \multicolumn{4}{|c|}{1 smoothing step } & \multicolumn{4}{|c|}{2 smoothing steps } & \multicolumn{4}{|c|}{4 smoothing steps } \\
\hline & \multicolumn{2}{|c|}{$\mathbf{u w} p$} & \multicolumn{2}{|c|}{$\mathbf{u v} p$} & \multicolumn{2}{|c|}{$\mathbf{u w} p$} & \multicolumn{2}{|c|}{$\mathbf{u v} p$} & \multicolumn{2}{|c|}{$\mathbf{u w} p$} & \multicolumn{2}{|c|}{$\mathbf{u v} p$} \\
\hline & Iter. & $\mathrm{CPU}$ & Iter. & $\mathrm{CPU}$ & Iter. & $\mathrm{CPU}$ & Iter. & $\mathrm{CPU}$ & Iter. & $\mathrm{CPU}$ & Iter. & $\mathrm{CPU}$ \\
\hline 2 & 14 & 0.6 & Div. & Div. & 8 & 0.6 & 14 & 1.1 & 5 & 0.7 & 5 & 0.9 \\
\hline 3 & 16 & 3.7 & Div. & Div. & 8 & 3.9 & 20 & 8.9 & 4 & 4.0 & 5 & 4.3 \\
\hline 4 & 17 & 17.4 & Div. & Div. & 8 & 16.9 & 15 & 30.3 & 5 & 19.1 & 5 & 19.1 \\
\hline \multicolumn{13}{|c|}{ time step size $=1 \times 10^{-3}[\mathrm{~s}]$} \\
\hline \multirow{3}{*}{ Level } & \multicolumn{4}{|c|}{1 smoothing step } & \multicolumn{4}{|c|}{2 smoothing steps } & \multicolumn{4}{|c|}{4 smoothing steps } \\
\hline & \multicolumn{2}{|c|}{$\mathbf{u w} p$} & \multicolumn{2}{|c|}{$\mathbf{u v} p$} & \multicolumn{2}{|c|}{$\mathbf{u w} p$} & \multicolumn{2}{|c|}{$\mathbf{u v} p$} & \multicolumn{2}{|c|}{$\mathbf{u w} p$} & \multicolumn{2}{|c|}{$\mathbf{u v} p$} \\
\hline & Iter. & $\mathrm{CPU}$ & Iter. & $\mathrm{CPU}$ & Iter. & $\mathrm{CPU}$ & Iter. & $\mathrm{CPU}$ & Iter. & $\mathrm{CPU}$ & Iter. & $\mathrm{CPU}$ \\
\hline 2 & 3 & 0.1 & Div. & Div. & 1 & 0.1 & 5 & 0.4 & 1 & 0.2 & 1 & 0.2 \\
\hline 3 & 3 & 0.7 & Div. & Div. & 2 & 0.8 & 3 & 1.3 & 1 & 1.0 & 1 & 1.0 \\
\hline 4 & 4 & 4.4 & Div. & Div. & 2 & 5.6 & 5 & 10.4 & 2 & 6.9 & 2 & 7.4 \\
\hline
\end{tabular}

\section{RESULTS FOR THE LINEAR CASE}

The three numerical examples, already solved in the first part of our work (see [17]) using the highly accurate $\mathbf{u v} p(3)-\mathrm{TR}-\mathrm{Q} 2 / \mathrm{P} 1$ solver, were solved again but with our new $\mathbf{u w} p-\mathrm{TR}-\mathrm{Q} 2 / \mathrm{P} 1$ solver, which stands for the described monolithic solver for the uw $p$ formulation based on the weak forms (42)-(45) using the implicit Crank-Nicolson $(\theta=1 / 2)$ time integration scheme as shown in (48)-(53) and the mixed finite element pairs Q2/P1 shown in Figure 1.

For the full description of the solver see Algorithm 2. By comparing the solutions of the past $\mathbf{u v} p$ (3)-TR-Q2/P1 for all problems of [17] with the new $\mathbf{u w} p-\mathrm{TR}-\mathrm{Q} 2 / \mathrm{P} 1$, we conclude that they both (1) have almost identical accuracy, (2) do not exhibit pressure instabilities and (3) show much weaker deficiencies at permeable loaded boundaries. In particular for large permeabilities, these deficiencies are not existent anymore.

And more interesting is that such oscillations (used to form a hindrance that deprives from using the more convenient boundary conditions, we are now using) did not show up despite of proceeding from the momentum balance of the whole mixture. This issue was discussed in detail in [5].

Next, we will study the efficiency of the special multigrid solver discussed in [17] for the new uw $p(3)-T R-Q 2 / P 1$ method. As example, the unstructured mesh of the large-scale problem presented in [17] is considered and based on Table I, it turned out that the new uw $p$ solver (in particular, in case of strong coupling) is remarkably better than the old $\mathbf{u v} p$ solver because the new converges even with one smoothing step while the old needs at least 2 smoothing steps to converge. 


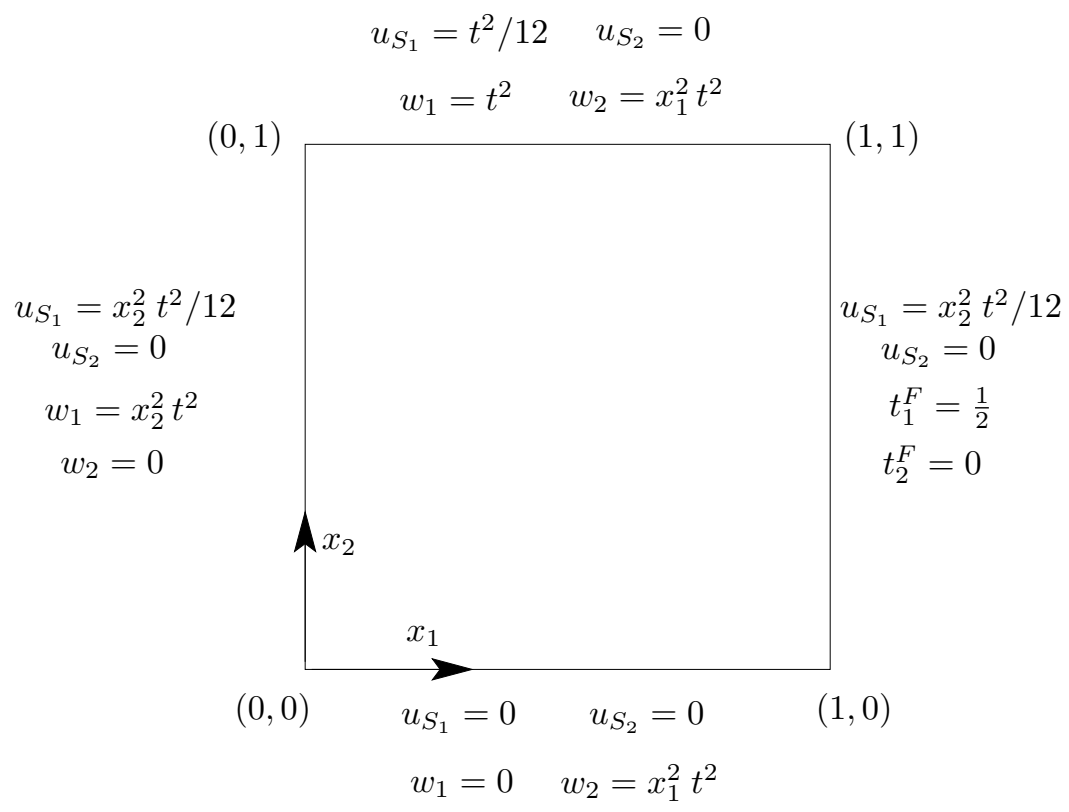

Figure 2. A square domain meshed with one element for mesh level 1. uw $p$-Q2/P1-TR was used to solve the problem. This problem has no real physical meaning and the UL formulation is deactivated to allow for pure testing of the Picard iterative method.

Table II. Physical properties of the porous medium used only for section 7 The gravitational acceleration is set to $10\left[\mathrm{~m} / \mathrm{s}^{2}\right]$

\begin{tabular}{|l|c|c|c|}
\hline Parameter & Symbol & Value & SI Unit \\
\hline second Lamé constant of solid & $\mu^{S}$ & 1 & {$\left[\mathrm{~N} / \mathrm{m}^{2}\right]$} \\
\hline first Lamé constant of solid & $\lambda^{S}$ & 1 & {$\left[\mathrm{~N} / \mathrm{m}^{2}\right]$} \\
\hline Effective density of dense solid & $\rho^{S R}$ & 2 & {$\left[\mathrm{~kg} / \mathrm{m}^{3}\right]$} \\
\hline Effective density of pore fluid & $\rho^{F R}$ & 1 & {$\left[\mathrm{~kg} / \mathrm{m}^{3}\right]$} \\
\hline Initial volume fraction of solid & $n_{0 S}^{S}$ & 0.5 & - \\
\hline Initial volume fraction of fluid & $n_{0 S}^{F}$ & 0.5 & - \\
\hline Initial permeability & $k_{0 S}^{F}$ & 1 & {$[\mathrm{~m} / \mathrm{s}]$} \\
\hline Permeability exponent & $\kappa$ & 1 & - \\
\hline
\end{tabular}

\section{SIMULATION OF ANALYTIC TEST PROBLEM}

Since we do not have yet results for a rigorous quantitative benchmark to compare with, we first of all present results for an analytical solution. This is a pure mathematical test which has no physical meaning. The UL formulation is deactivated (i. e., step 4 of Algorithm 1 is omitted) since $\mathbf{u}_{S}$ in this simulation does not indicate displacements that have a real physical meaning and are merely a mathematical function. The purpose is to validate the code and to make sure that the implementation of the Picard method, the time integrators, the generation of linear and bilinear forms and the implementation of the boundary conditions were done correctly by evaluating the L2and H1-norms of the error. The stress tensor $\mathbf{T}_{E}^{S}$ is defined in the appendix and the constant physical parameters are given in Table 
$u_{S_{1}}=\frac{1}{12} x_{2}^{2} t^{2}, \quad u_{S_{2}}=0, \quad w_{1}=x_{2}^{2} t^{2}, \quad w_{2}=x_{1}^{2} t^{2}, \quad p=\frac{1}{2}-x_{1}$

Then, the following set of equations were solved analytically:

$$
\begin{gathered}
\left(\rho \mathbf{v}_{S}\right)_{S}^{\prime}+\rho^{F R}(\mathbf{w})_{S}^{\prime}+\rho^{F R} \operatorname{grad}\left(\mathbf{v}_{F}\right) \mathbf{w}-\operatorname{div} \mathbf{T}_{E}^{S}+\operatorname{grad} p \\
-\rho^{F R}\left(\frac{n^{S}}{n^{F}}\right) \mathbf{w} \operatorname{div}\left(\mathbf{v}_{S}\right)+\left(\rho^{S R}-\rho^{F R}\right) n^{S} \operatorname{div}\left(\mathbf{v}_{S}\right) \mathbf{v}_{S}=\mathbf{f}_{u}, \\
\rho^{F R}\left(\mathbf{v}_{S}\right)_{S}^{\prime}+\rho^{F R}\left(\frac{\mathbf{w}}{n^{F}}\right)_{S}^{\prime}+\frac{\rho^{F R}}{n^{F}} \operatorname{grad}\left(\mathbf{v}_{F}\right) \mathbf{w}+\frac{\gamma^{F R}}{k^{F}} \mathbf{w}+\operatorname{grad} p=\mathbf{f}_{w}, \\
\operatorname{div}\left(\mathbf{v}_{S}\right)+\operatorname{div}(\mathbf{w})=0,
\end{gathered}
$$

where

$$
\begin{aligned}
& \mathbf{f}_{\mathrm{u}}=\left(\begin{array}{c}
\left(4 \mathrm{x}_{2} \mathrm{x}_{1}^{2}\right) \mathrm{t}^{4}+\left(\frac{1}{3} \mathrm{x}_{2} \mathrm{x}_{1}^{2}\right) \mathrm{t}^{3}-\left(\frac{1}{6}\right) \mathrm{t}^{2}+\left(2 \mathrm{x}_{2}^{2}\right) \mathrm{t}+\left(\frac{1}{4} \mathrm{x}_{2}^{2}-1\right) \\
\left(4 \mathrm{x} \mathrm{x}_{2}^{2}\right) \mathrm{t}^{4}+\left(2 \mathrm{x}_{1}^{2}\right) \mathrm{t}
\end{array}\right), \\
& \mathbf{f}_{w}=\left(\begin{array}{c}
\left(8 \mathrm{x}_{2} \mathrm{x}_{1}^{2}\right) \mathrm{t}^{4}+\left(\frac{2}{3} \mathrm{x}_{2} \mathrm{x}_{1}^{2}\right) \mathrm{t}^{3}+\left(10 \mathrm{x}_{2}^{2}\right) \mathrm{t}^{2}+\left(4 \mathrm{x}_{2}^{2}\right) \mathrm{t}+\left(\frac{1}{6} \mathrm{x}_{2}^{2}-1\right) \\
\left(8 \mathrm{x} \mathrm{x}_{2}^{2}\right) \mathrm{t}^{4}+\left(10 \mathrm{x}_{1}^{2}\right) \mathrm{t}^{2}+\left(4 \mathrm{x}_{1}^{2}\right) \mathrm{t}
\end{array}\right) .
\end{aligned}
$$

The domain, the boundary conditions and the mesh are depicted in Figure 2 Solving the problem analytically gives: It should be noted that the above solution functions are polynomials of second degree (for $\mathbf{u}_{S}$ and $\mathbf{w}$ ) and first degree (for $p$ ) which belong to the finite element space of the adopted Q2/P1 element pair ( 2 2 for $\mathbf{u}_{S}$ and $\mathbf{w}$, and P1 for $p$ ), thence using a one element mesh must be enough to eliminate the spatial errors. This allows us to focus on the time errors without being disturbed by spatial errors. The purpose of this problem is to check whether reduction in time of order 1 and 2 for BE and CN, respectively, does occur as it is supposed to do.

To this end, the FE solutions of $\mathbf{u w} p$-TR-Q2/P1 are directly compared with the analytical solutions via L2 and $\mathrm{H} 1$ norms of the errors as shown in Table III.

Next, we focus on spatial errors by picking an extremely small time step such that the temporal errors are almost non-existent. For this purpose, the same set of equations with the same physical parameters are solved but for the following right hand side functions:

$$
\begin{aligned}
& \mathbf{f}_{\mathrm{u}}=\left(\begin{array}{c}
\left(\frac{1}{12}\right) \mathrm{t}^{2}+\left(\mathrm{x}_{2}^{3}+\frac{1}{8} \mathrm{x}_{2}^{2}-1\right) \\
0
\end{array}\right), \\
& \mathbf{f}_{w}=\left(\begin{array}{c}
\left(10 \mathrm{x}_{2}^{3}\right) \mathrm{t}+\left(2 \mathrm{x}_{2}^{3}+\frac{1}{12} \mathrm{x}_{2}^{2}-1\right) \\
0
\end{array}\right) .
\end{aligned}
$$

The domain, the boundary conditions and the mesh are depicted in Figure 3 .

Solving the problem analytically for the full assumption gives the following exact solution functions: The results are shown in table IV 
Table III. The errors in the finite element solutions at time $t=0.01[\mathrm{~s}]$ and mesh level 1 of the ULP-uw $p$ Q2/P1 solver for both Crank Nicolson (CN) and Backward Euler (BE) measured through the L2 and H1 norms.

\begin{tabular}{|c|c|c|c|}
\hline \multirow{2}{*}{ error norm } & \multicolumn{3}{|c|}{ Backward Euler (BE) } \\
\hline & $\Delta \mathrm{t}=1 \times 10^{-5}[\mathrm{~s}]$ & $\Delta \mathrm{t}=1 \times 10^{-6}[\mathrm{~s}]$ & $\Delta \mathrm{t}=1 \times 10^{-7}[\mathrm{~s}]$ \\
\hline$\| \mathbf{u}_{\mathrm{S}}-$ ref. $\|_{\mathrm{L} 2}$ & $2.0 \times 10^{-9}$ & $2.0 \times 10^{-10}$ & $2.0 \times 10^{-11}$ \\
\hline$\left\|\mathbf{v}_{\mathrm{S}}-\mathrm{ref} .\right\|_{\mathrm{L} 2}$ & $2.6 \times 10^{-8}$ & $2.6 \times 10^{-9}$ & $2.6 \times 10^{-10}$ \\
\hline$\| \mathbf{w}-$ ref. $\|_{\mathrm{L} 2}$ & $3.4 \times 10^{-8}$ & $3.4 \times 10^{-9}$ & $3.4 \times 10^{-10}$ \\
\hline$\| p-$ ref. $\|_{\mathrm{L} 2}$ & $2.8 \times 10^{-6}$ & $2.8 \times 10^{-7}$ & $2.8 \times 10^{-8}$ \\
\hline$\| \mathbf{u}_{\mathrm{S}}-$ ref. $\|_{\mathrm{H} 1}$ & $9.0 \times 10^{-9}$ & $9.0 \times 10^{-10}$ & $9.0 \times 10^{-11}$ \\
\hline$\| \mathbf{v}_{\mathrm{S}}-$ ref. $\|_{\mathrm{H} 1}$ & $1.2 \times 10^{-7}$ & $1.2 \times 10^{-8}$ & $1.2 \times 10^{-9}$ \\
\hline$\| \mathbf{w}-$ ref. $\|_{\mathrm{H} 1}$ & $1.5 \times 10^{-7}$ & $1.5 \times 10^{-8}$ & $1.5 \times 10^{-9}$ \\
\hline$\| \mathrm{p}-$ ref. $\|_{\mathrm{H} 1}$ & $6.3 \times 10^{-6}$ & $6.3 \times 10^{-7}$ & $6.3 \times 10^{-8}$ \\
\hline \multirow{2}{*}{ error norm } & \multicolumn{3}{|c|}{ Crank Nicolson $(\mathrm{CN})$} \\
\hline & $\Delta \mathrm{t}=1 \times 10^{-4}[\mathrm{~s}]$ & $\Delta \mathrm{t}=1 \times 10^{-5}[\mathrm{~s}]$ & $\Delta \mathrm{t}=1 \times 10^{-6}[\mathrm{~s}]$ \\
\hline$\| \mathbf{u}_{\mathrm{S}}-$ ref. $\|_{\mathrm{L} 2}$ & $9.3 \times 10^{-11}$ & $9.3 \times 10^{-13}$ & $9.3 \times 10^{-15}$ \\
\hline$\left\|\mathbf{v}_{\mathrm{S}}-\mathrm{ref} .\right\|_{\mathrm{L} 2}$ & $3.4 \times 10^{-17}$ & $3.5 \times 10^{-17}$ & $3.7 \times 10^{-17}$ \\
\hline$\| \mathbf{w}-$ ref. $\|_{\text {L2 }}$ & $1.6 \times 10^{-9}$ & $1.6 \times 10^{-11}$ & $1.6 \times 10^{-13}$ \\
\hline$\| \mathrm{p}-$ ref. $\|_{\mathrm{L} 2}$ & $1.7 \times 10^{-14}$ & $1.2 \times 10^{-14}$ & $5.7 \times 10^{-14}$ \\
\hline$\| \mathbf{u}_{\mathrm{S}}-$ ref. $\|_{\mathrm{H} 1}$ & $2.4 \times 10^{-10}$ & $2.4 \times 10^{-12}$ & $2.4 \times 10^{-14}$ \\
\hline$\| \mathbf{v}_{\mathrm{S}}-$ ref. $\|_{\mathrm{H} 1}$ & $1.5 \times 10^{-16}$ & $1.5 \times 10^{-16}$ & $1.6 \times 10^{-16}$ \\
\hline$\| \mathbf{w}-$ ref. $\|_{\mathrm{H} 1}$ & $4.1 \times 10^{-9}$ & $4.1 \times 10^{-11}$ & $4.1 \times 10^{-13}$ \\
\hline$\| \mathrm{p}-$ ref. $\|_{\mathrm{H} 1}$ & $4.7 \times 10^{-14}$ & $3.9 \times 10^{-14}$ & $1.4 \times 10^{-13}$ \\
\hline
\end{tabular}

$$
u_{S_{1}}=\frac{1}{24} x_{2}^{3} t, \quad u_{S_{2}}=0, \quad w_{1}=x_{2}^{3} t, \quad w_{2}=0, \quad p=\frac{1}{2}-x_{1} .
$$

Table IV. The errors in the finite element solutions for different mesh levels at time $t=0.01$ [s] of the ULPuw $p$-Q2/P1 solver for $\Delta t=1 \times 10^{-5}[\mathrm{~s}]$ for both Crank Nicolson and Backward Euler measured through the $\mathrm{L} 2$ and $\mathrm{H} 1$ norms.

\begin{tabular}{|c|c|c|c|c|}
\hline errors & mesh level 2 & mesh level 3 & mesh level 4 & reduction, level 3/ 4 \\
\hline$\| \mathbf{u}_{\mathrm{S}}-$ ref. $\|_{\mathrm{L} 2}$ & $1.81132 \times 10^{-6}$ & $2.27358 \times 10^{-7}$ & $2.82564 \times 10^{-8}$ & 8.0463 \\
\hline$\| \mathbf{v}_{\mathrm{S}}-$ ref. $\|_{\mathrm{L} 2}$ & $1.81207 \times 10^{-4}$ & $2.27381 \times 10^{-5}$ & $2.82476 \times 10^{-6}$ & 8.0496 \\
\hline$\| \mathbf{w}-$ ref. $\|_{\mathrm{L} 2}$ & $4.36619 \times 10^{-5}$ & $5.41021 \times 10^{-6}$ & $6.74468 \times 10^{-7}$ & 8.0214 \\
\hline$\| \mathrm{p}-$ ref. $\|_{\mathrm{L} 2}$ & $6.11228 \times 10^{-6}$ & $3.35809 \times 10^{-7}$ & $8.77083 \times 10^{-8}$ & 3.8287 \\
\hline$\| \mathbf{u}_{\mathrm{S}}-$ ref. $\|_{\mathrm{H} 1}$ & $2.33429 \times 10^{-5}$ & $5.86860 \times 10^{-6}$ & $1.46165 \times 10^{-6}$ & 4.0150 \\
\hline$\| \mathbf{v}_{\mathrm{S}}-$ ref. $\|_{\mathrm{H} 1}$ & $2.33538 \times 10^{-3}$ & $5.86971 \times 10^{-4}$ & $1.46140 \times 10^{-4}$ & 4.0165 \\
\hline$\| \mathbf{w}-$ ref. $\|_{\mathrm{H} 1}$ & $5.64424 \times 10^{-4}$ & $1.40021 \times 10^{-4}$ & $3.49498 \times 10^{-5}$ & 4.0063 \\
\hline$\| \mathrm{p}-$ ref. $\|_{\mathrm{H} 1}$ & $2.33453 \times 10^{-5}$ & $1.68093 \times 10^{-6}$ & $4.95731 \times 10^{-7}$ & 3.3908 \\
\hline
\end{tabular}




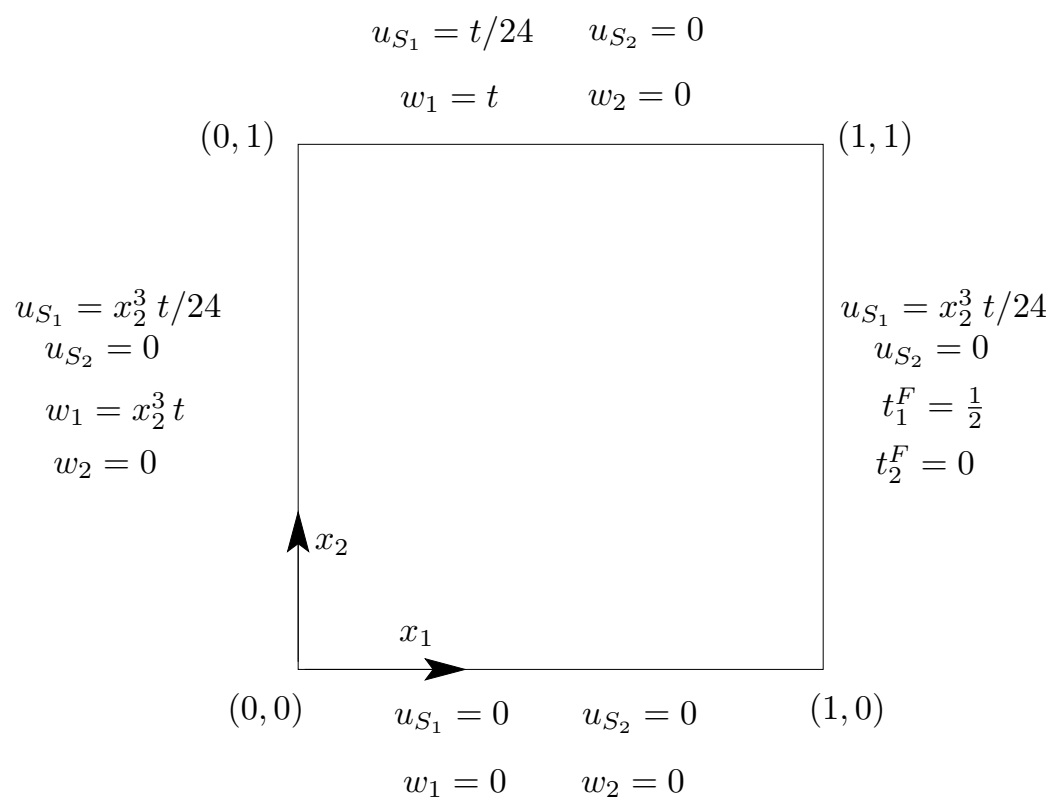

Figure 3. A square domain meshed with one element for mesh level 1. uw $p$-Q2/P1-TR was used to solve the problem. This problem has no real physical meaning and the UL formulation is deactivated to allow for pure testing of the Picard iterative method.

Table V. Physical properties of the porous medium used for all simulations except the ones in section7 The gravitational acceleration is set to $10\left[\mathrm{~m} / \mathrm{s}^{2}\right]$

\begin{tabular}{|l|c|c|c|}
\hline Parameter & Symbol & Value & SI Unit \\
\hline first Lamé constant of solid & $\mu^{S}$ & $5.583 \times 10^{6}$ & {$\left[\mathrm{~N} / \mathrm{m}^{2}\right]$} \\
\hline second Lamé constant of solid & $\lambda^{S}$ & $8.375 \times 10^{6}$ & {$\left[\mathrm{~N} / \mathrm{m}^{2}\right]$} \\
\hline Effective density of dense solid & $\rho^{S R}$ & 2000 & {$\left[\mathrm{~kg} / \mathrm{m}^{3}\right]$} \\
\hline Effective density of pore fluid & $\rho^{F R}$ & 1000 & {$\left[\mathrm{~kg} / \mathrm{m}^{3}\right]$} \\
\hline Initial volume fraction of solid & $n_{0 S}^{S}$ & 0.67 & - \\
\hline Darcy permeability & $k_{0 S}^{F}$ & from $10^{-5}$ to 0.5 & {$[\mathrm{~m} / \mathrm{s}]$} \\
\hline
\end{tabular}

\section{TWO-DIMENSIONAL WAVE PROPAGATION IBVP}

In this example, we study the 2D dynamical problem, depicted in Figure 4 under plane-strain conditions. The solid skeleton is hyper-elastic as defined in Section 2.3 and the appendix. The material parameters are given in Table $\mathbf{D}$.

The load $f(t)$ is given by

$$
f(t)=\sin (25 \pi t)[1-H(t-\tau)]\left[10^{6} \mathrm{~Pa}\right]
$$

with $H(t-\tau)$ being the Heaviside step function and $\tau=0.04$ [s]. The water saturated mixture domain is surrounded by impermeable, frictionless $\left(\bar{t}_{1}^{F}=0\right.$ for the bottom and $\bar{t}_{2}^{F}=0$ for the left and right sides) but rigid boundaries except for the loaded top side, which is perfectly drained $\left(\overline{\mathbf{t}}^{F}=\mathbf{0}\right)$. The objective of this initial boundary value problem (IBVP) is to study quantitatively the effect of the volume fractions rate of change (the green terms in (38) ) and the effect of convection (the orange terms in (38)-(39) ) on the solutions. Here we differentiate between three reduced cases: (1) 'fully reduced' in which all the green terms and all the orange terms are neglected, (2) 'no orange' that excludes only the convection, (3) 'no green' case where only the changes in volume 


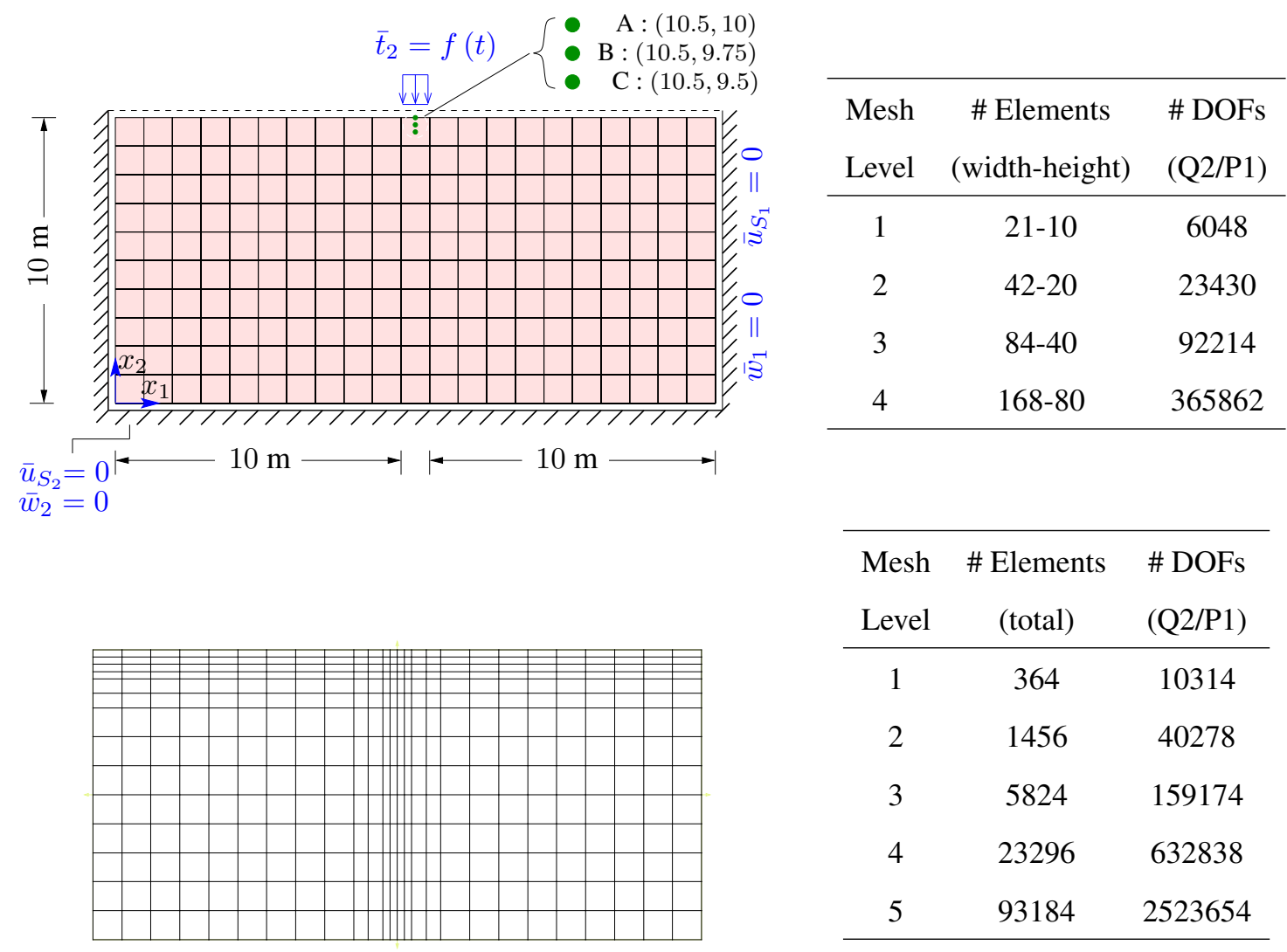

Figure 4. Geometry, boundary conditions and isotropic mesh level 1 of the symmetric 2D wave propagation problem (top-left). Total number of elements and unknowns for the Q2/P1 approach (top-right). Anisotropic mesh level 1 (bottom-left). total number of elements and unknowns for the Q2/P1 approach (bottomright). Going from mesh level $\mathrm{i}$ to mesh level $\mathrm{i}+1$, every old local element is isotropically refined into 4 new elements. The symmetry of the problem can be exploited to reduce the problem size. However, the computation was performed for the full problem.

fractions are ignored. To do so, we prefer to do the comparison on a mesh level that leads to full convergence of the solutions $\left(u_{S_{2}}\right.$ and $p$ ) for the full $\mathbf{u w} p$ formulation. Therefore, three equidistant points initially located on the axis of symmetry and in the first half meter below the top loaded boundary were opted for this purpose. The results are depicted in Figure 5] and show the full convergence on mesh level 5.

Based on the results of this problem (for sample solutions, see Figure 6), we observed that the convection may noticeably influence the fluidic solution components $\left(p\right.$ and $\left.\mathbf{v}_{F}\right)$ if the considered $k_{S}^{F}$ is large enough. However, the influence on the deformation $\left(\mathbf{u}_{S}\right)$ by convection remains negligible. On the other hand, the influence on the pressure is much weaker than the deformation in case of volume fraction changes (the green terms) and remains very small for both $\mathbf{u}_{S}$ and $p$. To gain a better picture, the deviations of the solutions $p_{r}$ (subscript $r$ refers to one of three reduced cases) from the solutions $p$ of the 'full ' $\mathbf{u w} p$ form is quantitatively measured using the following relative error formula

$$
\operatorname{error}_{p}=\frac{\sum_{i=1}^{n}\left|p\left(t_{i}\right)-p_{r}\left(t_{i}\right)\right|}{\sum_{i=1}^{n}\left|p\left(t_{i}\right)\right|},
$$

where $n$ denotes the number of time steps. By replacing $p$ in (63) with $u_{S_{2}}$, we obtain the formula for error $_{u 2}$. 
Point A

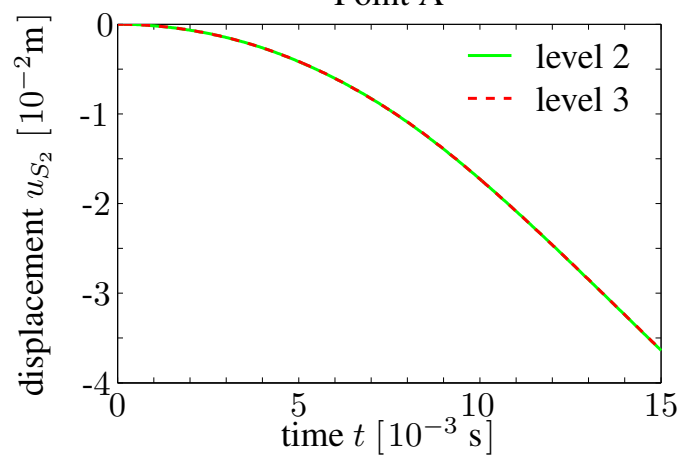

Point B
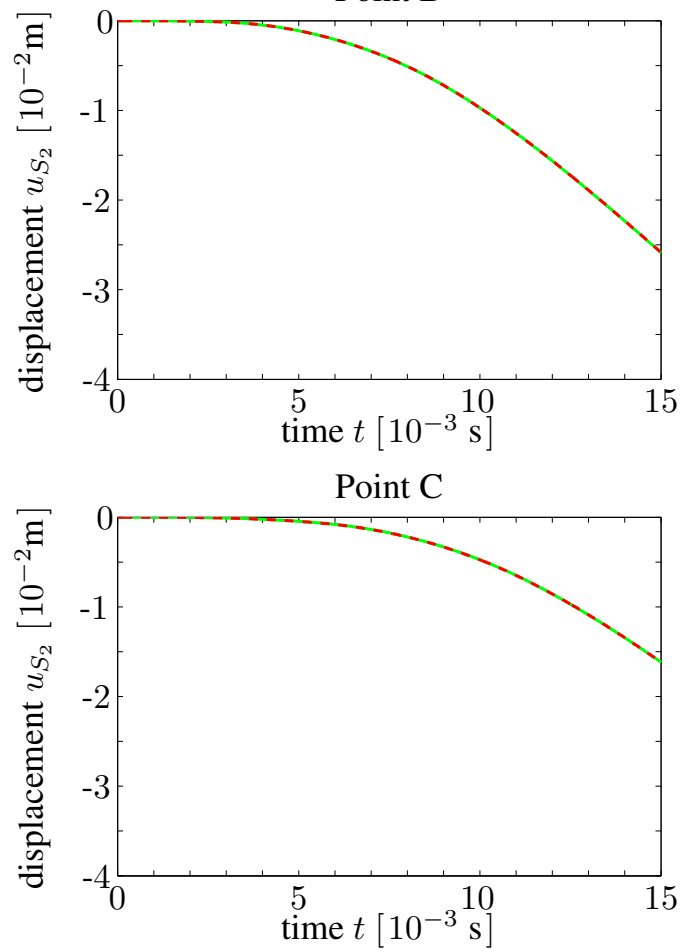

Point A

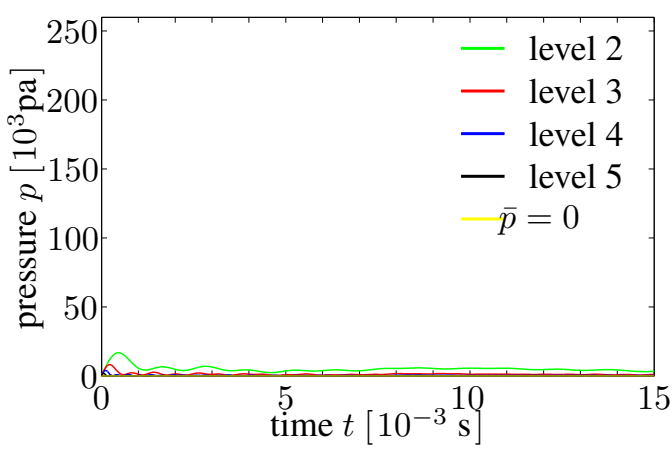

Point B

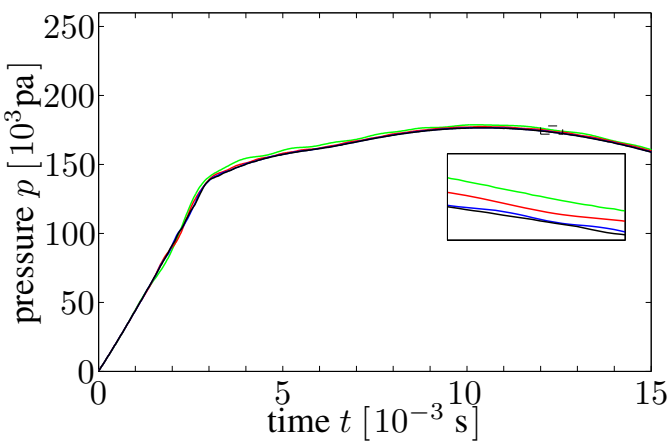

Point C

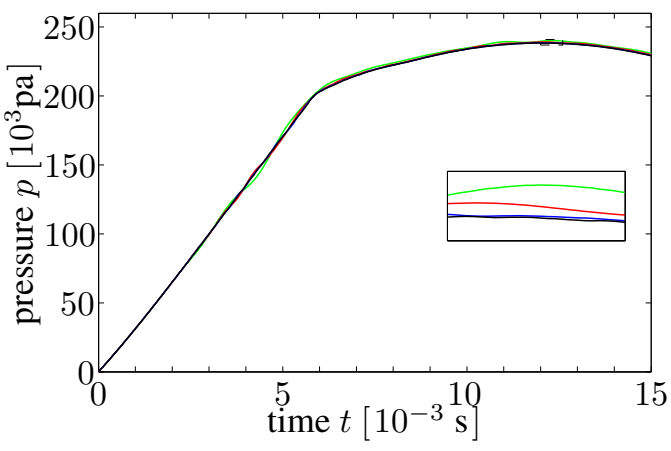

Figure 5. The displacement and the pressure versus time for different points located on the axis of symmetry in the first meter below the top loaded boundary for $k_{0 S}^{F}=10^{-2}[\mathrm{~m} / \mathrm{s}], \kappa=1$ and for the three reduced cases using ULP-uw $p$-TR-Q2/P1 method with $\Delta t=2.5 \times 10^{-5}[\mathrm{~s}]$ and the anisotropic mesh level 5.

Based on Figure 7 we conclude that in case of strong coupling: (i. e., moderately small $k^{F}$ $\left(k^{F} \leq 10^{-5}[\mathrm{~m} / \mathrm{s}]\right)$ and very small $\left.k^{F}\left(k^{F} \leq 10^{-10}[\mathrm{~m} / \mathrm{s}]\right)\right)$, the convective terms become more or less unimportant and can be canceled, whereas in case of weak coupling it depends on our main concern. That is, if we are only interested in the deformation $\mathbf{u}_{S}$, then the convectiveless assumption is not bad, otherwise, the convection should be involved.

‡Note that by use of (2), $n^{F}$ in 21 , can be factored out and then for $\kappa=1$, we have that $\frac{n^{\mathrm{F}} \gamma^{\mathrm{FR}}}{k^{\mathrm{F}}}=\frac{n_{0}^{\mathrm{F}} \gamma^{\mathrm{FR}}}{k_{0 S}^{\mathrm{F}}}=$ const. $\times \frac{1}{k_{0 S}^{\mathrm{F}}}$ which means that the fraction remains constant with time and hence, we can study its effect by switching the values of $k_{0 S}^{\mathrm{F}}$ only. 

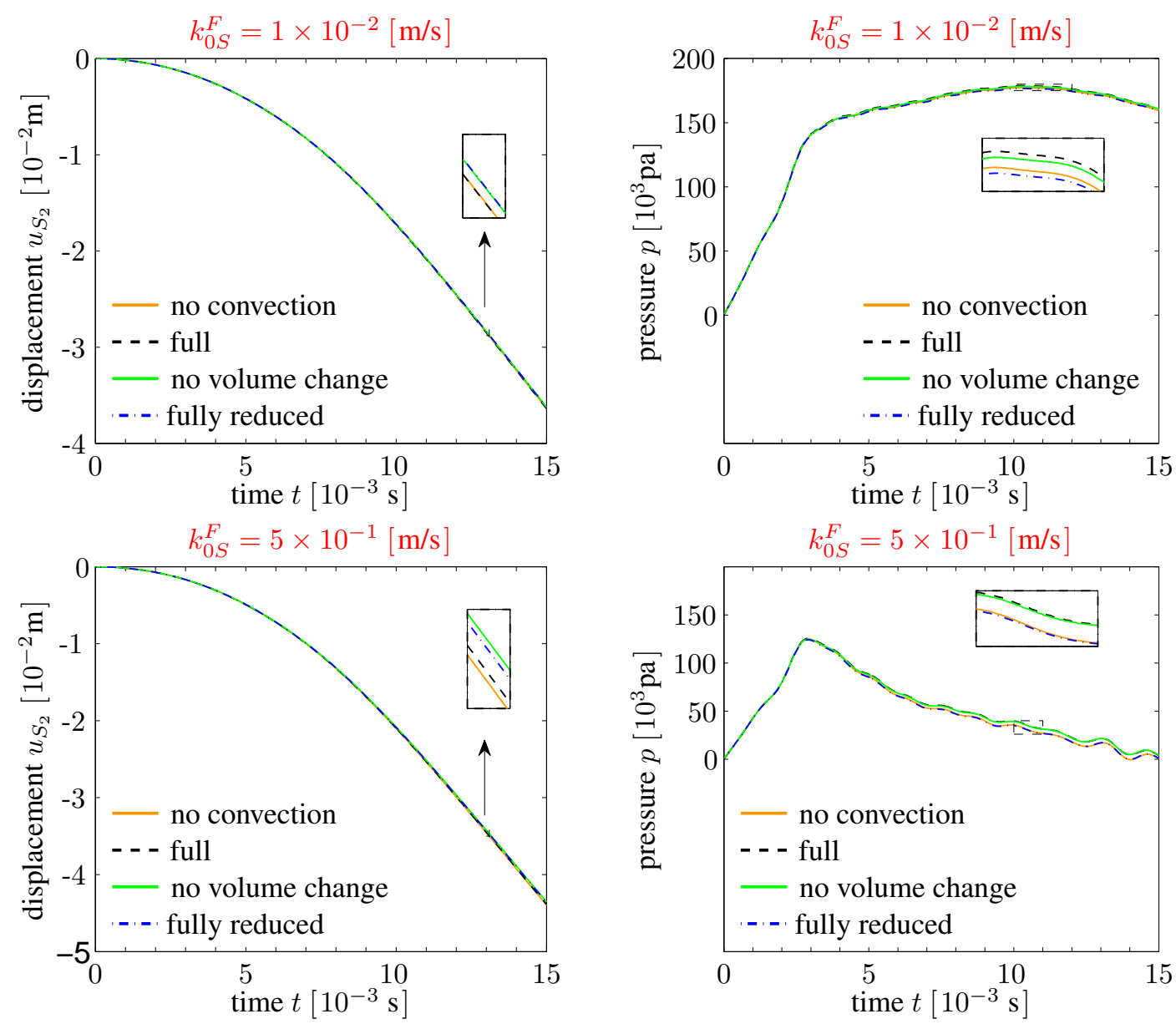

Figure 6. The displacement and the pressure at a point $\mathrm{B}$ for $k_{0 S}^{F}=10^{-2}[\mathrm{~m} / \mathrm{s}], \kappa=1$ and for the three reduced cases using ULP-uw $p$-TR-Q2/P1 method with $\Delta t=2.5 \times 10^{-5}[\mathrm{~s}] \mathrm{s}$ and the anisotropic mesh level 5 .

\section{ADAPTIVE TIME STEPPING (ATS)}

The convergence speed of the ULP solver is strongly influenced by the non-linear Cauchy extra stress $\mathbf{T}_{E}^{S}$ which, in turn, is inversely proportional to the minimum value of the deformation dependent $n^{F}$. This can be seen if we let $n^{F} \rightarrow 0$. Then by (8) $J \rightarrow n_{0 S}^{S}$ and consequently $\tilde{h}\left(J_{\mathrm{S}}\right)$ in (69) goes to infinity.

Strictly speaking, if $\min _{\mathbf{x} \in \Omega} n^{F}(\mathbf{x})$ gets smaller, then corresponding local $\mathbf{T}_{E}^{S}$ becomes larger and more iterations are required. However, when integrating in time, the non-linear operator $\operatorname{div} \mathbf{T}_{E}^{S}$ is drastically reduced by small $\Delta t$ as shown below,

$$
\begin{aligned}
\rho \mathbf{v}_{S}+\rho^{F R} \mathbf{w}+\theta \Delta t \rho^{F R} \operatorname{grad} \mathbf{v}_{F} \mathbf{W} & -\theta \Delta t \operatorname{div} \mathbf{T}_{E}^{S}-\theta \Delta t(\rho)_{S}^{\prime} \mathbf{v}_{S} \\
& -\theta \Delta t \frac{\left(\rho^{F}\right)_{S}^{\prime}}{n^{F}} \mathbf{W}+\operatorname{grad} \tilde{p}=\mathbf{r h s}
\end{aligned}
$$

so that $\Delta t \operatorname{div} \mathbf{T}_{E}^{S}$ is not dominant over the weakly non-linear momentum terms.

A particular $\Delta t$ which means to lower the dominant non-linear term $\mathbf{T}_{E}^{S}$ may not be suitable if $\mathbf{T}_{E}^{S}$ raises above a certain limit and as a result, we need to switch to another smaller $\Delta t$. Therefore, using fixed time steps is not recommended here. 

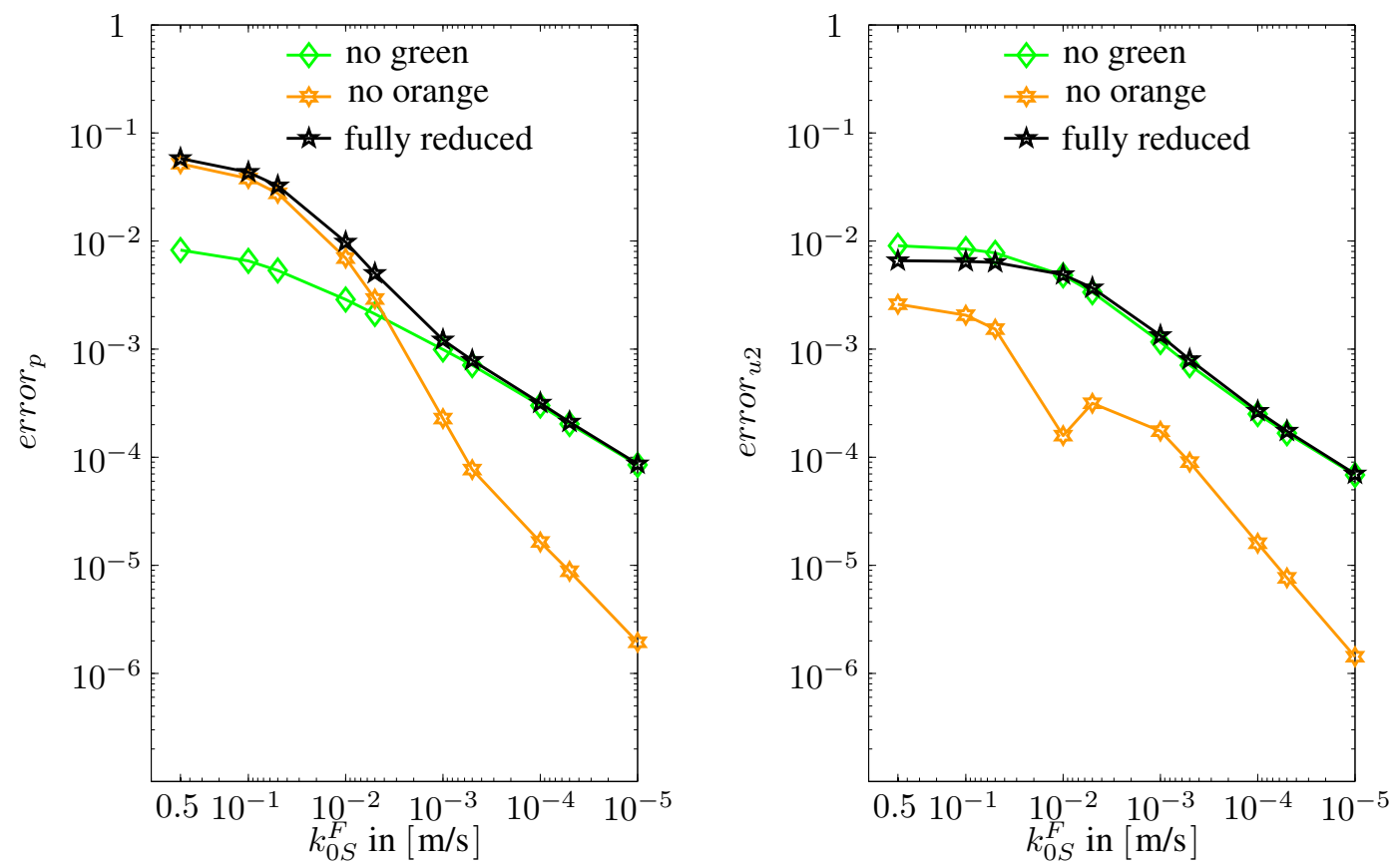

Figure 7. error $_{p}$ and error $_{u 2}$ computed using (63) at a point initially located at the axis of symmetry and $0.25 \mathrm{~m}$ below the top loaded boundary for $\kappa=1$ and for the three reduced cases using ULP-uw $p$-TR-Q2/P1 method with $\Delta t=2.5 \times 10^{-5} \mathrm{~s}$ and the anisotropic mesh level 5 .

To demonstrate this fact, we will adopt the problem of a saturated poroelastic column (cf. Figure 8) under compression load, because for this specific problem, we know that

$$
\min _{\mathbf{x} \in \Omega} n^{F}(\mathbf{x}(t))=n^{F}\left(\mathbf{x}_{\text {top }}(t)\right) \quad \forall t \in[02],
$$

where $\mathbf{x}_{t o p}$ refers to the top surface.

Note from Figure 9, we frequently have to shorten the time step size $\Delta t$ to adapt to the further increase in the non-linear $\mathbf{T}_{E}^{S}$, provoked by smaller $\mathrm{n}^{\mathrm{F}}\left(\mathbf{x}_{\mathrm{top}}\right)$ in $t \in[02]$.

But this reduction in $\Delta t$ should be done carefully so that the simulation is finished as fast as possible and within the desired accuracy. An excessive reduction in the time step size will prolong the CPU time, while poor reduction may slow down the speed of convergence of the ULP. Therefore, a possibility for enlarging the time step size to accompany weakening non-linearity that may occur must be considered.

Since we do not yet have an excellent predictor for the best time step increase or decrease, we present an adaptive time stepping algorithm (see Algorithm 3) which differs from Algorithm 1 by the red statements.

The algorithm uses the non-linear convergence rate $\xi$ for iteration $i$,

$$
\xi^{i}=\sqrt[i]{\frac{\left\|\mathbf{d}^{i}\right\|}{\left\|\mathbf{d}^{0}\right\|}},
$$

as indicator for the strength of the non-linearity to adjust the time step size.

Here, we set an upper bound $\left(\xi_{\max }\right)$ for $\xi^{i}$ and if $\xi^{i}$ happens to exceed $\xi_{\max }$, the time step gets aborted (unless the solver accidental converged to the desired tolerance to avoid time wasting) and then reduced by rat\% as described in the algorithm.

Large values for percentage reduction rat\% produce highly oscillating $\Delta t$ 's, while low values yield almost smooth $\Delta t$ 's as shown in Figure 10 . 

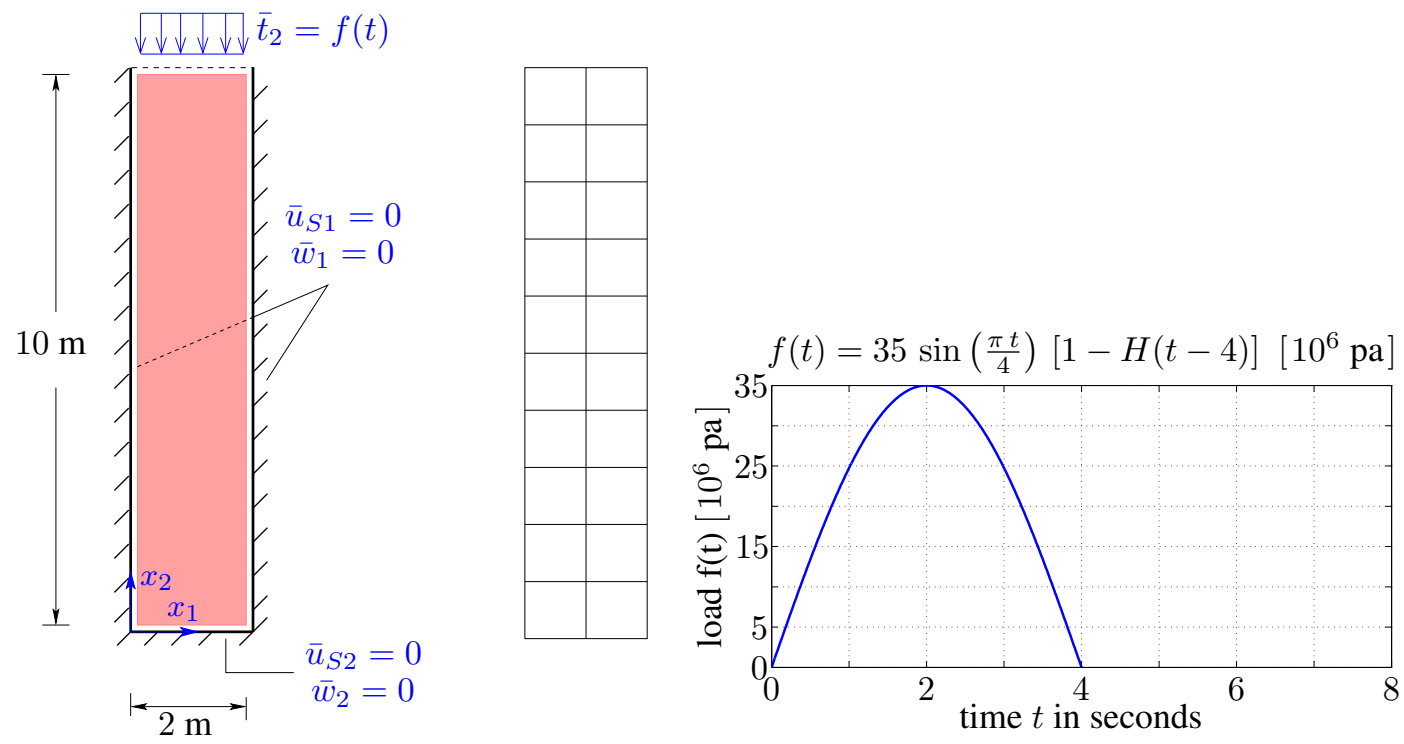

Figure 8. Geometry and boundary conditions (left) and Cartesian mesh level 1 with 1 element per meter (middle). The physical parameters are found in Table $\square$ and we set $k_{0 S}^{F}=10^{-4} \mathrm{~m} / \mathrm{s}$.
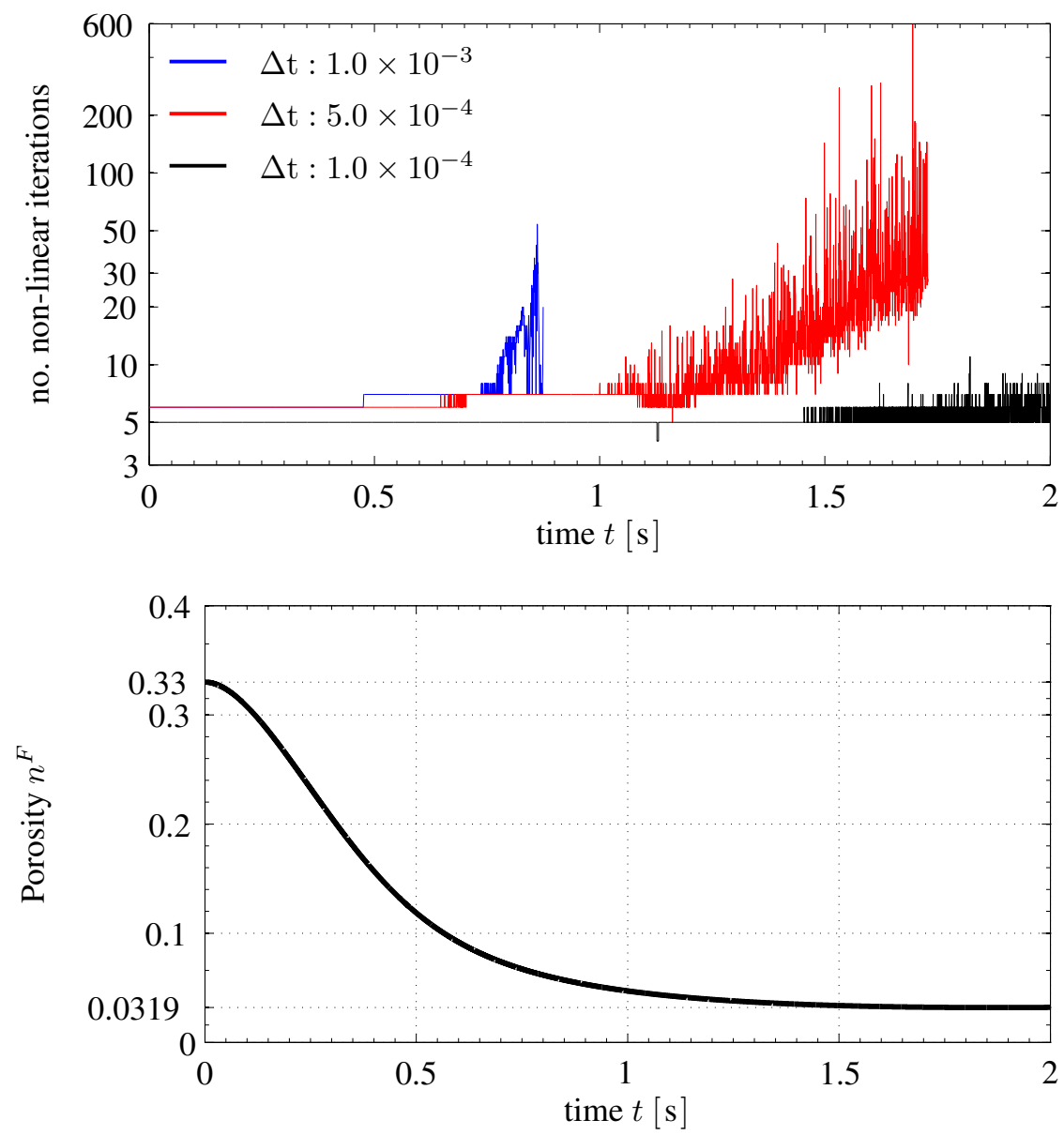

Figure 9. Number of non-linear iterations vs. time for ATS-fully-reduced-uw $p$-TR-Q2/P1-ULP-UMFPACK, mesh level 1 , tolerance $=10^{-5}$. 


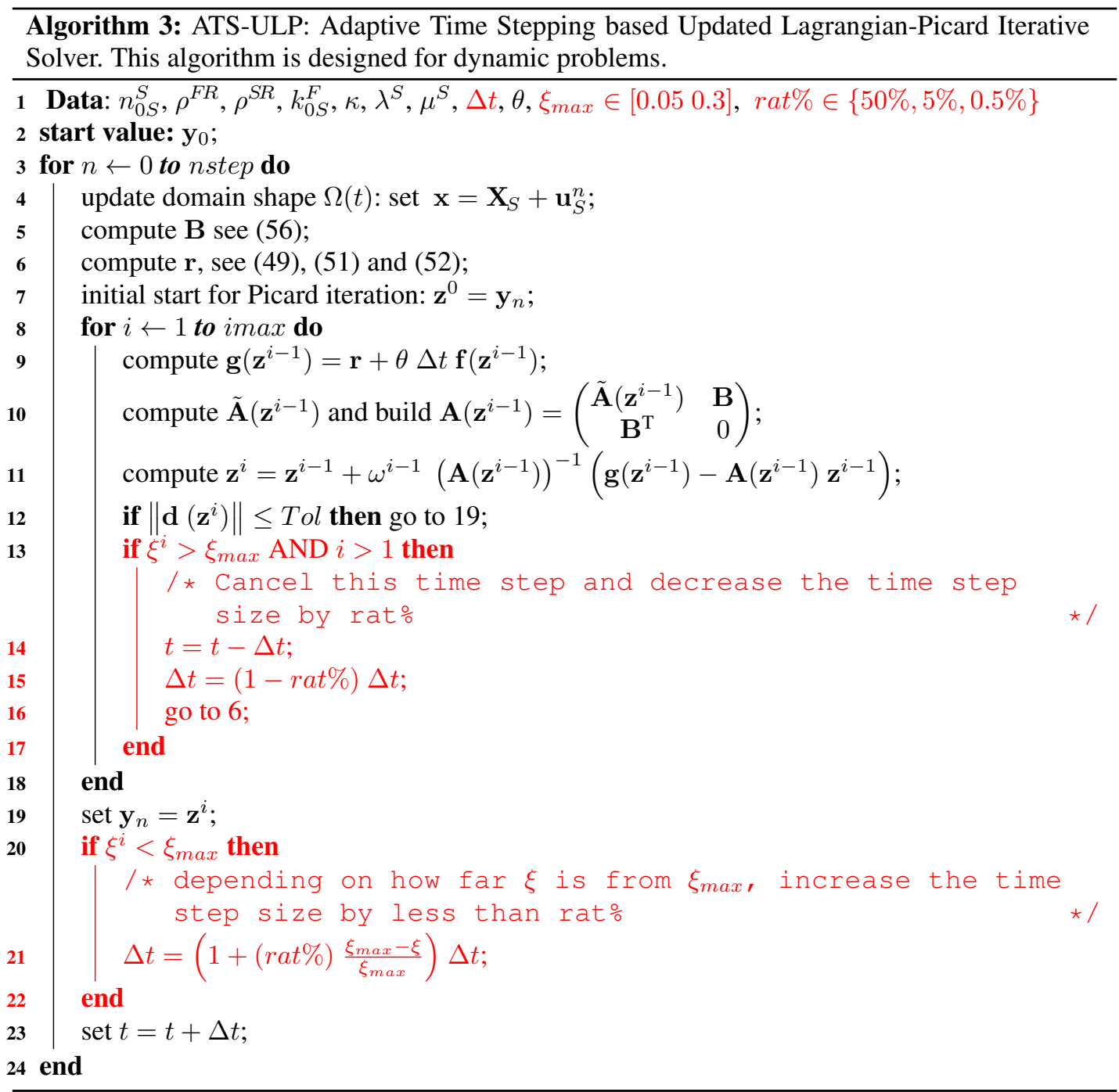

With regard to CPU timings, Table $\nabla 1$ shows similar values for $\operatorname{rat} \% \in\{50 \%, 5 \%, 0.5 \%\}$. Therefore, we shall switch to larger problems to find good combinations of $\xi_{\max }$ and $\mathrm{rat} \%$.

To do so, we will solve the problem illustrated in Figure 4, but with the following large impulse load

$$
f(t)=6.0 \sin (25 \pi t)[1-H(t-0.04)]\left[10^{6} \mathrm{pa}\right],
$$

which generates large compression (and hence large local stresses $\mathbf{T}_{E}^{S}$ ) quickly, such that fixed time steps may fail (or become impractical) and ATS is pressingly needed. Next, we inspect the point positioned on the intersection of the top boundary with the axis of symmetry because the axis of symmetry experiences the largest settling (or minimum $n^{F}$ ) for the considered time interval $\mathrm{t} \in\left[\begin{array}{ll}0 & 0.2\end{array}\right]$.

From Figure 11, we notice larger settling (and hence larger local $\mathbf{T}_{E}^{S}$ ), which requires a smaller $\Delta t$ and vice versa.

Now, we come to our main issue, that is finding good combinations $\xi_{\max }$ and $\mathrm{rat} \%$. From Table VII and Table VIII and since all $\xi_{\max } \leq 0.3$ give good accuracy, we conclude that $\xi_{\max } \in\left[\begin{array}{ll}0.05 & 0.3]\end{array}\right.$ (in particular $\xi_{\max }=0.1$ ) together with rat $=5 \%$ are pretty good choices.

It remains to mention that in order to get the best $\Delta t$ generated by this procedure (that is, a $\Delta t$ which is as large as possible), one needs to start with an initial $\Delta t$ large enough (for instance $3 \times 10^{-3}$ for our problems), so that it gets reduced from first the time step because otherwise, the 

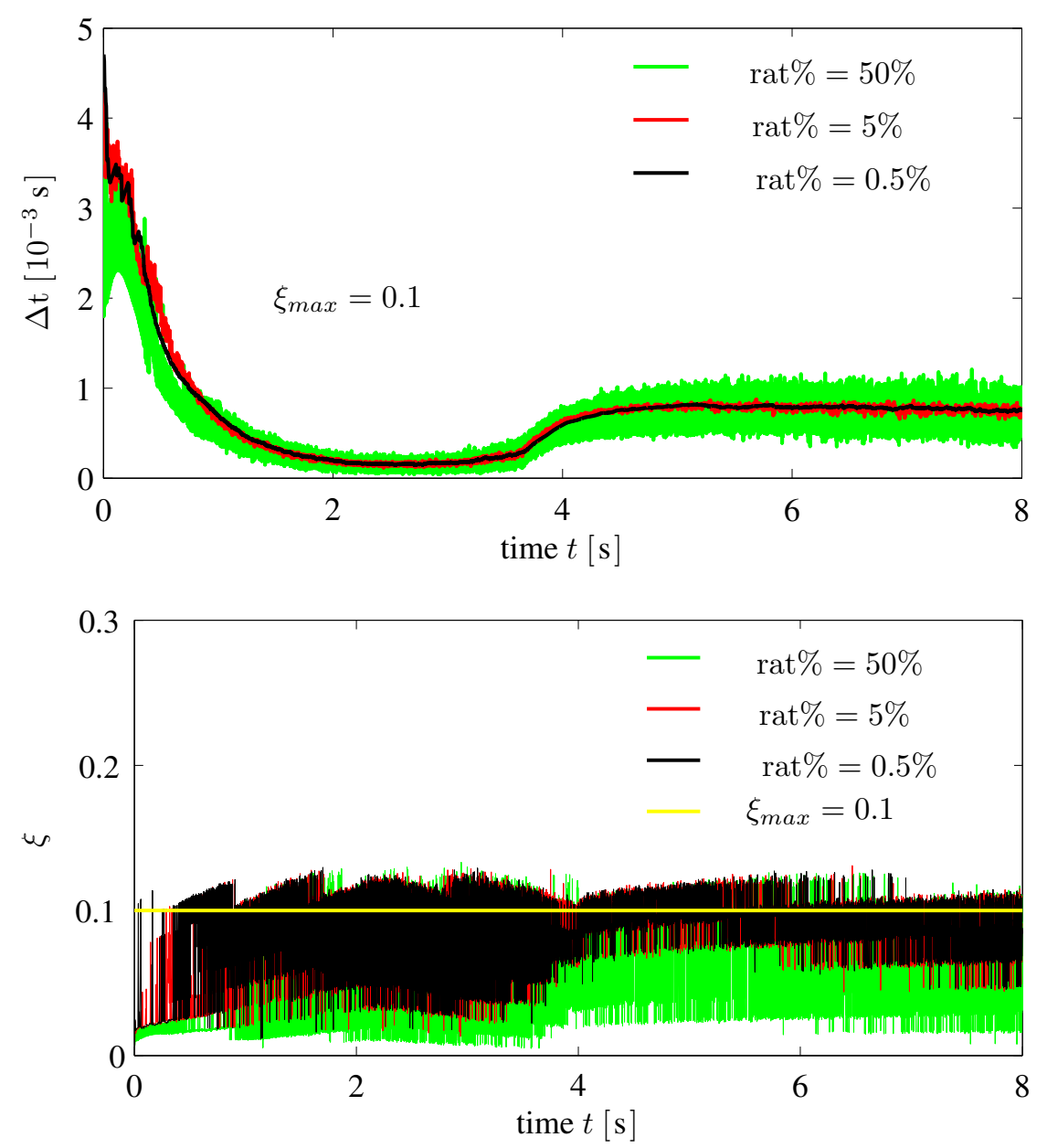

Figure 10. Time step $\Delta t$ vs. time (left) and $\xi$ vs. time (right) for fully-reduced-uw $p$-TR-Q2/P1, mesh level 1 , tolerance $=10^{-12}$.

selected $\Delta t$ may be too small and the small rat $\%$ increase will take long time to reach to the optimum option for this technique.

\section{CONCLUSION}

In preparation for the numerical treatment, the governing equations of porous media dynamics were briefly recapped. This covers the porous media modeling approach, the corresponding kinematics as well as the equilibrium and constitutive relations. Mathematically, this leads eventually to a set of four non-linear elliptic partial differential equations, which need to be solved: (1) the balance of momentum of the solid phase, (2) the balance of momentum of the fluid phase, (3) the volume balance of the mixture, (4) the solid velocity-displacement relation.

Thereafter, the governing set of these four PDEs was converted into Stokes-like form, referred to as uw $p$ formulation. The purpose is to permit the direct application of some special CFD techniques, used to solve the incompressible Navier-Stokes equation and already realized in our in-house code FEATFLOW2.

The discretization in time has been done by the standard $\theta$-scheme, while the spatial discretization has been carried out by the well-known (non-parametric) Q2/P1 finite element pair, which belongs 
Table VI. Total number of steps (\# Step), Total number of non-linear iterations (\# Iter.) and total elapsed CPU time (CPU) in seconds for the described ULP solver for $t \in\left[\begin{array}{ll}0 & 2\end{array}\right] \mathbf{u w} p$-TR-Q2/P1 + UMFPACK including the successful and the aborted steps.

\begin{tabular}{|c|c|c|c|c|c|c|}
\hline \multicolumn{7}{|c|}{ rat $\%=0.5 \%$} \\
\hline$\xi_{\text {max }}$ & \# level 1 & level 2 \\
& \# Step & \# Iter & CPU & \# Step & \# Iter & CPU \\
\hline 0.01 & 209346 & 782538 & 9332 & 338114 & 1598911 & 158058 \\
\hline 0.05 & 27131 & 209857 & 2252 & 159781 & 1048473 & 99478 \\
\hline 0.10 & 23788 & 243543 & 2585 & 138671 & 1135668 & 110425 \\
\hline 0.30 & 16896 & 304417 & 3217 & 109966 & 1520808 & 143824 \\
\hline \multicolumn{7}{|c|}{ rat\% $\% 5 \%$} \\
\hline$\xi_{\text {max }}$ & \# Step & \# Iter & CPU & \# Step & \# Iter & CPU \\
\hline 0.01 & 211895 & 785829 & 9188 & 340414 & 1610024 & 159398 \\
\hline 0.05 & 26944 & 208853 & 2340 & 161031 & 1053202 & 99742 \\
\hline 0.10 & 23623 & 239730 & 2531 & 139526 & 1134378 & 109241 \\
\hline 0.30 & 15704 & 290501 & 2992 & 110735 & 1512872 & 140950 \\
\hline \multicolumn{7}{|c|}{ level 1 } \\
\hline$\xi_{\text {max }}$ & \# Step & \# Iter & CPU & \# Step & \# Iter & CPU \\
\hline 0.01 & 256174 & 950533 & 11088 & 382596 & 1714069 & 170020 \\
\hline 0.05 & 29073 & 203776 & 2204 & 184107 & 1136921 & 111305 \\
\hline 0.10 & 26692 & 241811 & 2588 & 159349 & 1193064 & 107856 \\
\hline 0.30 & 17711 & 289811 & 3013 & 127108 & 1509608 & 139835 \\
\hline
\end{tabular}

to the best FEM choices for incompressible flow problems in terms of efficiency, accuracy and robustness. The outcome is a weak form that demands less regularity and allows Neumann boundary conditions that are more convenient to apply.

The resulting systems of non-linear algebraic equations were solved by means of the updated Lagrange-Picard (ULP) method, which does not entail computations of time-consuming material tangent matrix and many other Gateaux derivatives.

For every non-linear iteration, either the direct UMFPACK solver or iterative fast geometrical multigrid method with special block Vanka smoother (both are subroutines already implemented in FEATFLOW) are called to precondition the non-linear residual generated by ULP.

The previously mentioned special CFD approach $(\mathrm{Q} 2 / \mathrm{P} 1$ FE pair $+\mathbf{u w} p$ formulation $+\theta$-scheme + special multigrid + ULP) was then validated through several analytical simulations via L2and H1-error norms. However, only two of them were recorded in this paper. After that, more realistic simulations were performed to investigate the effect of the convections and the volume changes on the final solutions. The final results were then provided. In conclusion and after several numerical test cases performed on canonical one and two-dimensional wave propagation examples, we strongly recommend our new CFD approach, which is not only highly accurate, but also speeds up the special multigrid solver, demands less regularity, does not require the computation of the expensive material tangent matrix, involves easy-to-impose solution-independent boundary 

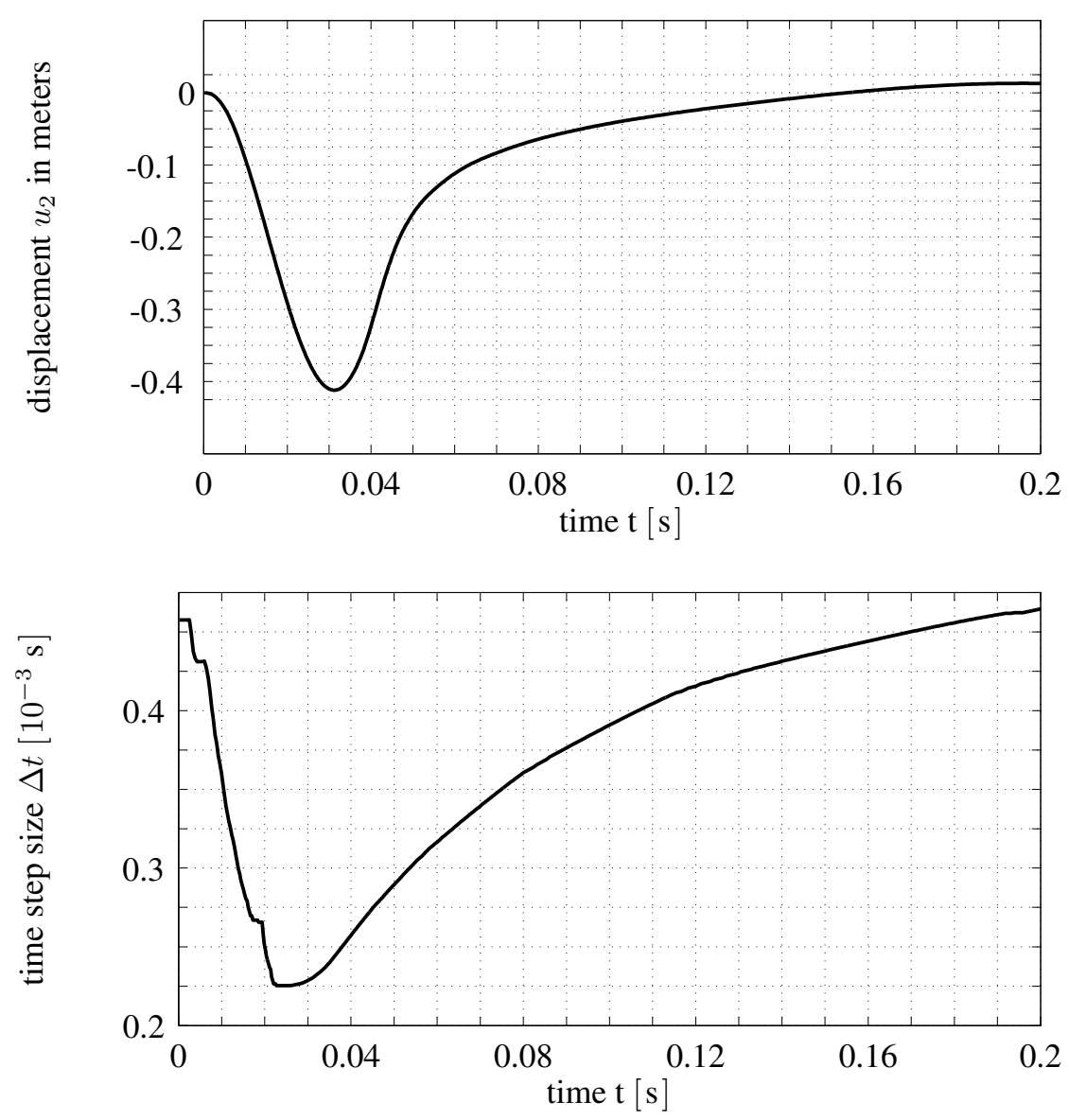

Figure 11. Time step $\Delta t$ vs. time (right) Displacement of point A vs. time (left). The results were generated for uw $p$-TR-Q2/P1-ATS-ULP-UMFPACK solver with $r a t \%=0.5 \%$ and $\xi_{\max }=0.1$, mesh level 2 , tolerance $=10^{-12}$ and $k_{0 S}^{F}=10^{-2}[\mathrm{~m} / \mathrm{s}]$.

conditions and typical for the most challenging situation, general three field non-linear PMD problem with strong coupling and with no restriction to the considered frequency range in which the reduced $\mathbf{u} p$ form is no longer feasible. Finally, an adaptive time stepping strategy was suggested to cope with situations in which the strength of non-linearity (or number of non-linear iterations per time step) may suddenly (and rapidly) go up or down.

In future works, we will investigate the effect of the material tangent matrix on the performance of this approach and account for different material models. Moreover, an extension of our implementation to 3D problems is on the road map, which opens the avenue to more practically relevant applications

\section{APPENDIX}

We shall show how to compute $\mathbf{K}_{\mathbf{u u}}$ and $\mathbf{h}$ mentioned in (42). Recall that the Cauchy extra stress tensor $\mathbf{T}_{E}^{S}$ for the considered hyper-elastic material is given by the material model

$$
\mathbf{T}_{E}^{S}=\frac{\mu^{S}}{J_{\mathrm{S}}}\left(\mathbf{F}_{S} \mathbf{F}_{S}^{\mathrm{T}}-\mathbf{I}\right)+\tilde{h}\left(J_{\mathrm{S}}\right) \mathbf{I},
$$


Table VII. Total number of steps (\# Step), Total number of non-linear iterations (\# Iter.) and total elapsed CPU time (CPU) in seconds for the described ATS-ULP solver for $t \in\left[\begin{array}{ll}0 & 0.2\end{array}\right]$ and convectiveless uw $p$-TRQ2/P1 + UMFPACK (as preconditioner) including the successful and the aborted steps. This table is related to the configuration of Figure 4 with load as defined in $(65)$.

\begin{tabular}{|c|c|c|c|c|c|c|}
\hline \multicolumn{7}{|c|}{$k_{0 S}^{F}=10^{-2}[\mathrm{~m} / \mathrm{s}]$} \\
\hline \multicolumn{7}{|c|}{ rat $\%=0.5 \%$} \\
\hline \multirow{2}{*}{$\xi_{\max }$} & \multicolumn{3}{|c|}{ level 1} & \multicolumn{3}{|c|}{ level 2} \\
\hline & \# Step & \# Iter & $\mathrm{CPU}$ & \# Step & \# Iter & $\mathrm{CPU}$ \\
\hline 0.01 & 2651 & 12114 & 2616 & 4117 & 21537 & 22383 \\
\hline 0.05 & 803 & 4508 & 960 & 1130 & 9641 & 9934 \\
\hline 0.10 & 609 & 4142 & 879 & 854 & 9182 & 9418 \\
\hline 0.30 & 413 & 3579 & 755 & 547 & 10240 & 10464 \\
\hline \multicolumn{7}{|c|}{ rat $\%=5 \%$} \\
\hline \multirow{2}{*}{$\xi_{\max }$} & \multicolumn{3}{|c|}{ level 1} & \multicolumn{3}{|c|}{ level 2} \\
\hline & \# Step & \# Iter & $\mathrm{CPU}$ & \# Step & \# Iter & $\mathrm{CPU}$ \\
\hline 0.01 & 1525 & 9090 & 1950 & 2942 & 17911 & 18556 \\
\hline 0.05 & 366 & 3526 & 741 & 712 & 7433 & 7664 \\
\hline 0.10 & 257 & 3124 & 657 & 514 & 6783 & 6958 \\
\hline 0.30 & 158 & 3244 & 674 & 314 & 7392 & 7551 \\
\hline \multicolumn{7}{|c|}{ rat $\%=50 \%$} \\
\hline \multirow{2}{*}{$\xi_{\max }$} & \multicolumn{3}{|c|}{ level 1} & \multicolumn{3}{|c|}{ level 2} \\
\hline & \# Step & \# Iter & $\mathrm{CPU}$ & \# Step & \# Iter & $\mathrm{CPU}$ \\
\hline 0.01 & 1375 & 8354 & 1794 & 3072 & 18996 & 19677 \\
\hline 0.05 & 341 & 3527 & 745 & 741 & 7720 & 7928 \\
\hline 0.10 & 242 & 3190 & 671 & 580 & 7347 & 7536 \\
\hline 0.30 & 159 & 3522 & 734 & 381 & 8015 & 8188 \\
\hline
\end{tabular}

where $\mu^{S}$ is the first lame parameter for solid skeleton, $\mathbf{F}_{S}$ is the solid deformation gradient and defined as follows

$$
\mathbf{F}_{S}^{-1}=\mathbf{I}-\operatorname{grad} \mathbf{u}_{S}
$$

and $J_{\mathrm{S}}$ is the solid Jacobian given by

$$
\frac{1}{J_{\mathrm{S}}}=\operatorname{det} \mathbf{F}_{S}^{-1}=1-\operatorname{div} \mathbf{u}_{S}+\left|\operatorname{grad} \mathbf{u}_{S}\right|
$$

for $2 \mathrm{D}$ problems. The source term $\tilde{h}\left(J_{\mathrm{S}}\right)$ is given by the following relation

$$
\tilde{h}\left(J_{\mathrm{S}}\right)=\lambda^{S}\left(1-n_{0 S}^{S}\right)^{2}\left(\frac{1}{1-n_{0 S}^{S}}-\frac{1}{J_{\mathrm{S}}-n_{0 S}^{S}}\right)
$$


Table VIII. Total number of steps (\# Step), Total number of non-linear iterations (\# Iter.) and total elapsed CPU time (CPU) in seconds for the described ATS-ULP solver for $t \in\left[\begin{array}{ll}0 & 0.2\end{array}\right]$ and convectiveless uw $p$-TR$\mathrm{Q} 2 / \mathrm{P} 1+\mathrm{UMFPACK}$ (as preconditioner) including the successful and the aborted steps. This table is related to the configuration of Figure 4 with load as defined in 65.

\begin{tabular}{|c|c|c|c|c|c|c|}
\hline \multicolumn{7}{|c|}{$k_{0 S}^{F}=10^{-5}[\mathrm{~m} / \mathrm{s}]$} \\
\hline$\xi_{\text {max }}$ & level 1 \\
& \# Step & \# Iter & CPU & \# Step & \# Iter & CPU \\
\hline 0.01 & 1192 & 6505 & 1417 & 2257 & 12852 & 14433 \\
\hline 0.05 & 666 & 4173 & 913 & 1127 & 8041 & 9107 \\
\hline 0.10 & 516 & 3638 & 787 & 877 & 7149 & 8086 \\
\hline 0.30 & 336 & 3484 & 744 & 582 & 6849 & 7697 \\
\hline \multicolumn{7}{|c|}{ rat\%=5\% $\%$} \\
\hline$\xi_{\text {max }}$ & \# Step & \# Iter & CPU & \# Step & \# Iter & CPU \\
\hline 0.01 & 688 & 5102 & 1106 & 1618 & 11090 & 12415 \\
\hline 0.05 & 318 & 3170 & 687 & 641 & 6602 & 7405 \\
\hline 0.10 & 232 & 2853 & 609 & 453 & 5789 & 6508 \\
\hline 0.30 & 140 & 2992 & 633 & 269 & 5861 & 6549 \\
\hline \multicolumn{7}{|c|}{ level 1 1} \\
\hline$\xi_{\text {max }}$ & \# Step & \# Iter & CPU & \# Step & \# Iter & CPU \\
\hline 0.01 & 660 & 5076 & 1098 & 1712 & 11990 & 13368 \\
\hline 0.05 & 288 & 3094 & 685 & 619 & 6662 & 7498 \\
\hline 0.10 & 228 & 2964 & 663 & 477 & 6272 & 7046 \\
\hline 0.30 & 150 & 3240 & 709 & 334 & 6785 & 7591 \\
\hline
\end{tabular}

Assuming that $\mathbf{T}_{E}^{S}=\left(\begin{array}{ll}T_{E_{11}}^{S} & T_{E_{12}}^{S} \\ T_{E_{21}}^{S} & T_{E_{22}}^{S}\end{array}\right)$ and $\mathbf{u}_{S}=\left(\begin{array}{l}u_{S_{1}} \\ u_{S_{2}}\end{array}\right)$, according to the previous 4 equations, it follows that

$$
\begin{aligned}
& T_{E_{11}}^{S}=\mu^{S}\left(1-u_{S_{2,2}}\right) u_{S_{1,1}}+\mu^{S}\left(J_{\mathrm{S}} u_{S_{1,2}}+u_{S_{2,1}}\right) u_{S_{1,2}}=h\left(J_{\mathrm{S}}\right) \\
&+\mu^{S}\left(J_{\mathrm{S}} u_{S_{2,2}}-2 J+1\right) u_{S_{2,2}}+\underbrace{\mu^{S}\left(J_{\mathrm{S}}-1\right)+\tilde{h}\left(J_{\mathrm{S}}\right)}, \\
& T_{E_{12}}^{S}=\mu^{S}\left(J_{\mathrm{S}}-J_{\mathrm{S}} u_{S_{1,1}}\right) u_{S_{1,2}}+\mu^{S}\left(J_{\mathrm{S}}-J_{\mathrm{S}} u_{S_{2,2}}\right) u_{S_{2,1}}, \\
& T_{E_{21}}^{S}=\mu^{S}\left(J_{\mathrm{S}}-J_{\mathrm{S}} u_{S_{1,1}}\right) u_{S_{1,2}}+\mu^{S}\left(J_{\mathrm{S}}-J_{\mathrm{S}} u_{S_{2,2}}\right) u_{S_{2,1}}, \\
& T_{E_{22}}^{S}=\mu^{S}\left(1-u_{S_{1,1}}\right) u_{S_{2,2}}+\mu^{S}\left(J_{\mathrm{S}} u_{S_{2,1}}+u_{S_{1,2}}\right) u_{S_{2,1}} \\
&+\mu^{S}\left(J_{\mathrm{S}} u_{S_{1,1}}-2 J+1\right) u_{S_{1,1}}+\underbrace{\mu^{S}\left(J_{\mathrm{S}}-1\right)+\tilde{h}\left(J_{\mathrm{S}}\right)} / .
\end{aligned}
$$


Where $u_{S_{i, j}}=\frac{\partial u_{S_{i}}}{\partial x_{j}}$ are the spatial derivates of the displacement and $\delta_{S_{i, j}}=\frac{\partial \delta S_{i}}{\partial x_{j}}$ are the spatial derivatives of the displacement test functions and they are both interpolated with the same basis function $\phi_{i}$. We have

$$
\int_{\Omega(t)} \operatorname{grad} \delta \mathbf{u}_{S}: \mathbf{T}_{E}^{S} \mathrm{~d} v=\left(\begin{array}{ll}
\delta u_{S_{1}} & \delta u_{S_{2}}
\end{array}\right) \underbrace{\left(\begin{array}{ll}
\mathbf{K}_{11} & \mathbf{K}_{12} \\
\mathbf{K}_{21} & \mathbf{K}_{22}
\end{array}\right)}_{\mathbf{K}_{\mathbf{u u}}}\left(\begin{array}{l}
u_{S_{1}} \\
u_{S_{2}}
\end{array}\right)+\left(\begin{array}{ll}
\delta u_{S_{1}} & \delta u_{S_{2}}
\end{array}\right) \underbrace{\left(\begin{array}{l}
h_{1} \\
h_{2}
\end{array}\right)}_{\mathbf{h}},
$$

where the element stiffness matrices are:

$$
\begin{aligned}
& \left(\mathbf{K}_{11}\right)_{i j}^{e}=\mu^{S} \int_{\Omega(t)}\left(1-u_{S_{2,2}}\right) \phi_{i, 1} \phi_{j, 1}+\left(J_{\mathrm{S}} u_{S_{1,2}}+u_{S_{2,1}}\right) \phi_{i, 1} \phi_{j, 2}+\left(J_{\mathrm{S}}-J_{\mathrm{S}} u_{S_{1,1}}\right) \phi_{i, 2} \phi_{j, 2} \mathrm{~d} v^{t}, \\
& \left(\mathbf{K}_{12}\right)_{i j}^{e}=\mu^{S} \int_{\Omega(t)}\left(J_{\mathrm{S}} u_{S_{2,2}}-2 J_{\mathrm{S}}+1\right) \phi_{i, 1} \phi_{j, 2}+\left(J_{\mathrm{S}}-J_{\mathrm{S}} u_{S_{2,2}}\right) \phi_{i, 2} \phi_{j, 1} \mathrm{~d} v^{t} \\
& \left(\mathbf{K}_{2,1}\right)_{i j}^{e}=\mu^{S} \int_{\Omega(t)}\left(J_{\mathrm{S}} u_{S_{1,1}}-2 J_{\mathrm{S}}+1\right) \phi_{i, 2} \phi_{j, 1}+\left(J_{\mathrm{S}}-J_{\mathrm{S}} u_{S_{1,1}}\right) \phi_{i, 1} \phi_{j, 2} \mathrm{~d} v^{t} \\
& \left(\mathbf{K}_{22}\right)_{i j}^{e}=\mu^{S} \int_{\Omega(t)}\left(1-u_{S_{1,1}}\right) \phi_{i, 2} \phi_{j, 2}+\left(J_{\mathrm{S}} u_{S_{2,1}}+u_{S_{1,2}}\right) \phi_{i, 2} \phi_{j, 1}+\left(J_{\mathrm{S}}-J_{\mathrm{S}} u_{S_{2,2}}\right) \phi_{i, 1} \phi_{j, 1} \mathrm{~d} v^{t}
\end{aligned}
$$

These element matrices are then assembled to give the global matrices $\mathbf{K}_{11}, \mathbf{K}_{12}, \mathbf{K}_{21}$ and $\mathbf{K}_{22}$. We also get extra source terms $\mathbf{h}$ due to the material model which consist of two components:

$$
\begin{aligned}
& \left(h_{1}\right)_{i}^{e}=\int_{\Omega(t)} \phi_{i, 1} \cdot h\left(J_{\mathrm{S}}\right) \mathrm{d} v^{t}=\mu^{S} \int_{\Omega(t)} \phi_{i, 1} \cdot\left(J_{\mathrm{S}}-1+\frac{\tilde{h}\left(J_{\mathrm{S}}\right)}{\mu^{S}}\right) \mathrm{d} v^{t}, \\
& \left(h_{2}\right)_{i}^{e}=\int_{\Omega(t)} \phi_{i, 2} \cdot h\left(J_{\mathrm{S}}\right) \mathrm{d} v^{t}=\mu^{S} \int_{\Omega(t)} \phi_{i, 2} \cdot\left(J_{\mathrm{S}}-1+\frac{\tilde{h}\left(J_{\mathrm{S}}\right)}{\mu^{S}}\right) \mathrm{d} v^{t} .
\end{aligned}
$$

The element source terms are then assembled to give the global sources $h_{1}$ and $h_{2}$.

\section{ACKNOWLEDGEMENTS}

We thank the FEATFLOW2 developers, Michael Köster and Peter Zajac, for the intensive support in the early stages of implementing the presented model into FEATFLOW2. Moreover, this work was partially supported by the German Research Foundation (DFG) under grant TU102/34.

\section{REFERENCES}

1. Markert B. A constitutive approach to 3-d nonlinear fluid flow through finite deformable porous continua. Transp. Porous Med. 2007; 70:427-450.

2. Markert B. A biphasic continuum approach for viscoelastic high-porosity foams: Comprehensive theory, numerics, and application. Arch. Comput. Methods Eng. 2008; 15:371-446.

3. Heider Y, Markert B, Ehlers W. Dynamic wave propagation in infinite saturated porous media half spaces. Computational Mechanics 2012; 49:319-336.

4. Heider Y, Avci O, Markert B, Ehlers W. The dynamic response of fluid-saturated porous materials with application to seismically induced soil liquefaction. Soil Dyn Earthq Eng 2014; 63:120-137.

5. Markert B, Heider Y, Ehlers W. Comparison of monolithic and splitting solution schemes for dynamic porous media problem. Int. J. Numer. Meth. Eng. 2010; 82:1341-1383.

6. Markert B. A survey of selected coupled multifield problems in computational mechanics. J Coupled Syst Multiscale Dyn 2013; 27:22-48.

7. de Boer R. Theory of Porous Media. Springer-Verlag, Berlin, 2000.

8. Ehlers W. Foundations of multiphasic and porous materials. Porous Media: Theory, Experiments and Numerical Applications, Ehlers W, Bluhm J (eds.). Springer-Verlag, Berlin, 2002; 3-86.

9. Zienkiewicz OC, Shiomi T. Dynamic behaviour of saturated porous media: the generalized Biot formulation and its numerical solution. Int. J. Numer. Anal. Methods Geomech. 1984; :71-96. 
10. Zienkiewicz OC, Chan AHC, Pastor M, Schrefler BA, Shiomi T. Computational geomechanics with special reference to earthquake engineering. Wiley, Chichester, 1999.

11. Diebels S, Ehlers W. Dynamic analysis of a fully saturated porous medium accounting for geometrical and material non-linearities. Int. J. Numer. Methods Eng. 1996; 39:81-97.

12. Breuer S. Quasi-static and dynamic behavior of saturated porous media with incompressible constituents. Transp. Porous Media 1999; 34:285-303.

13. de Boer R, Ehlers W, Liu Z. One-dimensional wave propagation in fluid saturated incompressible porous media. Arch. Appl. Mech. 1993; 63:59-72.

14. Schanz M. Transient wave propagation in a one-dimensional poroelastic column. Acta Mech. 2000; 145:1-18.

15. Felippa CA, Park KC, Farhat C. Partitioned analysis of coupled mechanical systems. Comput. Methods Appl. Mech. Engrg. 2001; 190:3247-3270.

16. Matthies HG, Niekamp R, Steindorf J. Algorithms for strong coupling procedures. Comput. Methods Appl. Mech. Engrg. 2006; 195:2028-2049.

17. Turek S, Obaid A, Markert B. On a fully implicit, monolithic finite element method-multigrid solution approach for dynamic porous media problems. Journal of Coupled Systems and Multiscale Dynamics 2013;

18. Ehlers W. Foundations of multiphasic and porous materials. Porous Media: Theory, Experiments and Numerical Applications, Ehlers W, Bluhm J (eds.). Contemporary Mathematics, Springer-Verlag Berlin Heidelberg, 2002; 3-86.

19. Ehlers W, Eipper G. Finite elastic deformations in liquid-saturated and empty porous solids. Transport in Porous Media 1999; 34(1-3):179-191, doi:10.1023/A:1006565509095.

20. Diebels S, Ehlers W, Markert B. Neglect of the fluid extra stresses in volumetrically coupled solid-fluid problems. ZAMM 2001; 81:521 - 522.

21. Turek S. Efficient solvers for incompressible flow problems: An algorithmic and computational approach. Springer, 1999.

22. Razzaq M, Damanik H, Hron J, Ouazzi A, Turek S. FEM multigrid techniques for fluid-structure interaction with application to hemodynamics. Applied Numerical Mathematics 2011; (62):1156-1170.

23. Turek S, Hron J, Madlik M, Razzaq M, Wobker H, Acker J. Numerical simulation and benchmarking of a monolithic multigrid solver for fluid-structure interaction problems with application to hemodynamics. FluidStructure Interaction II: Modelling, Simulation, Optimisation, Bungartz H, Mehl M, Schäfer M (eds.). Springer, 2010. Doi 10.1007/978-3-642-14206-2.

24. Turek S, Hron J, Razzaq M, Schäfer M. Numerical benchmarking of fluid-structure interaction: A comparison of different discretization and solution approaches. Fluid-Structure Interaction II: Modelling, Simulation, Optimisation, Bungartz H, Mehl M, Schäfer M (eds.). Springer, 2010.

25. Razzaq M, Turek S, Hron J, Damanik H, Ouazzi A. FEM multigrid techniques for fluid-structure interaction with application to hemodynamics. Applied Numerical Mathematics Oct 2012; 62(9):1156 - 1170. 